\title{
Appendix: On Edge-Isoperimetric Theorems for Uniform Hypergraphs
}

\author{
R. Ahlswede and N. Cai
}

\section{Introduction}

Denote by $\Omega=\{1, \ldots, n\}$ an $n$-element set. For all $A, B \in\left(\begin{array}{c}\Omega \\ k\end{array}\right)$, the $k$-element subsets of $\Omega$, define the relation $\sim$ as follows:

$A \sim B$ iff $A$ and $B$ have a common shadow, i.e. there is a $C \in\left(\begin{array}{c}\Omega \\ k-1\end{array}\right)$ with $C \subset A$ and $C \subset B$. For fixed integer $\alpha$, our goal is to find a family $\mathcal{A}$ of $k$-subsets with size $\alpha$, having as many as possible $\sim$-relations for all pairs of its elements. For $k=2$ this was achieved by Ahlswede and Katona [2] many years ago. However, it is surprisingly difficult for $k \geq 3$, in particular there is no complete solution even for $k=3$. Perhaps, the reason is the complicated behaviour for "bad $\alpha$ " so that the most natural and reasonable conjecture, which will be described in the last section and was mentioned already in [2], is false. Actually, our problem can also be viewed as a kind of isoperimetric problem in the sense of Bollobás and Leader ([4], see also [6]). They gave two versions. Partition the vertex set $V$ of a graph $G=(V, E)$ into 2 parts $A$ and $A^{c}$ such that for fixed $\alpha|A|=\alpha$ and

I. The subgraph induced by $A$ has maximal number of edges or

II. The number of edges connecting vertices from $A$ and $A^{c}$ is as small as possible.

When $G$ is regular, the two versions are equivalent. In our case we define $G=$ $(V, E)$ by $V=\left(\begin{array}{c}\Omega \\ k\end{array}\right)$ and $E=\{\{A, B\} \subset V: A \neq B$ and $A \sim B\}$. Thus the original problem is an edge-isoperimetric problem for a certain regular graph. In order

to solve our problem, in Section 2 we reduce it to another kind of problem, which we call "sum of ranks problem" : For a lattice with a rank function find a downset of given size with maximal sum of the ranks of its elements. Similar questions were studied in [3], [6], and [8]. In Section 3, we go over to a continuous version of the problem and solve it for $k=3$ and "good $\alpha$ ". Some of the auxiliary results and ideas there extend also to general $k$. A related but much simpler result concerning a moment problem is presented in Section 4.

\section{From Edge-Isoperimetric to Sum of Ranks Problem}

In this section we reduce the edge-isoperimetric problem to the sum of ranks problem. Denote by $\mathcal{L}(n, k)=\left(S_{n, k}, \leq\right)$ the lattice defined by 


$$
S_{n, k}=\left\{\left(x_{1}, \ldots, x_{k}\right): 1 \leq x_{1}<x_{2} \cdots<x_{k} \leq n, x_{i} \in \mathbb{Z}^{+}\right\}
$$

and $\left(x_{1}, \ldots, x_{k}\right) \leq\left(x_{1}^{\prime}, \ldots, x_{k}^{\prime}\right) \Leftrightarrow x_{i} \leq x_{i}^{\prime}(1 \leq i \leq k)$. For $x^{k} \in S_{n, k}$, the rank of $x^{k}$ is defined as $\left|x^{k}\right|=\sum_{i=1}^{k} x_{i}$ and for $W \subset S_{n, k}$, let $\|W\|=\sum_{x^{k} \in W}\left|x^{k}\right|$. In addition we let $A=\left\{x_{1}, \ldots, x_{k}\right\} \in\left(\begin{array}{c}\Omega \\ k\end{array}\right)$, with elements labelled in increasing order, correspond to $x^{k}=\Phi(A) \triangleq\left(x_{1}, \ldots, x_{k}\right) \in S_{n, k}$, and, similarly, $\mathcal{A} \subset\left(\begin{array}{l}\Omega \\ k\end{array}\right)$ to $\Phi(\mathcal{A})=\{\Phi(A): A \in \mathcal{A}\}$. Moreover, for $\mathcal{A} \subset\left(\begin{array}{l}\Omega \\ k\end{array}\right)$ we introduce

$$
\mathcal{P}(\mathcal{A})=\left\{(A, B) \in \mathcal{A}^{2}: A \sim B\right\} .
$$

Using for $A \in \mathcal{A}$ and $1 \leq i<j \leq n$ the following "pushing to the left" or so-called switching operator $O_{i, j}$, which is frequently employed in combinatorial extremal theory:

$$
O_{i, j}(A)=\underset{A}{(A \backslash\{j\}) \cup\{i\}} \text { if }(A \backslash\{j\}) \cup\{i\} \notin \mathcal{A}, j \in A, \text { and } i \notin A
$$

one can prove, by standard arguments, that for fixed $\alpha$ an $\mathcal{A} \subset\left(\begin{array}{l}\Omega \\ k\end{array}\right)$ with $|\mathcal{A}|=\alpha$, which maximizes $|\mathcal{P}(\mathcal{A})|$, can be assumed to be within a family of subsets, which are invariant under the pushing to left operator. It is also easy to see that such subsets correspond to a downset in $\mathcal{L}(n, k)$.

Lemma 1. For $\alpha \in \mathbb{Z}^{+} \max _{|\mathcal{A}|=\alpha}|\mathcal{P}(\mathcal{A})|$ is assumed by an $\mathcal{A} \subset\left(\begin{array}{c}\Omega \\ k\end{array}\right)$ s.t. $\Phi(\mathcal{A})$ is a downset in $\mathcal{L}(n, k)$.

Now we are ready to show the first of our main results.

Theorem 1. For fixed $\alpha \in \mathbb{Z}^{+}$, maximizing $|\mathcal{P}(\mathcal{A})|$ for $\mathcal{A} \subset\left(\begin{array}{c}\Omega \\ k\end{array}\right),|\mathcal{A}|=\alpha$, is equivalent to finding a downset $W$ in $\mathcal{L}(n, k)$ with $|W|=\alpha$ and maximal $\|W\|$.

Proof. Assume that $\mathcal{A} \subset\left(\begin{array}{c}\Omega \\ k\end{array}\right), W=\Phi(\mathcal{A})$ is a downset in $\mathcal{L}(n, k)$, and $|\mathcal{A}|=\alpha$.

For every $x^{k} \in W$ there are exactly

$$
\left(x_{i+1}-x_{i}-1\right)\left(\begin{array}{c}
k-i \\
k-1-i
\end{array}\right)=\left(x_{i+1}-x_{i}-1\right)(k-i)
$$

$y^{k}$ 's with $y^{k} \leq x^{k}$, whose first $i$ components coincide with those of $x^{k}$ and the $(i+1)$-st components differ, and for which $A$ and $B$ have a common shadow if $x^{k}=\Phi(A)$ and $y^{k}=\Phi(B)$. (Here $x_{0} \triangleq 0$.) By (1.1), for $x^{k}=\Phi(A)$ fixed, there is a total of

$$
\begin{gathered}
\sum_{i=0}^{k-1}\left(x_{i+1}-x_{i}-1\right)(k-i)=\sum_{i=1}^{k}(k-i+1) x_{i}-\sum_{i=0}^{k-1}(k-i) x_{i}-\sum_{i=0}^{k-1}(k-i) \\
=\sum_{i=1}^{k} x_{i}-\left(\begin{array}{c}
k+1 \\
2
\end{array}\right)=\left|x^{k}\right|-\left(\begin{array}{c}
k+1 \\
2
\end{array}\right)
\end{gathered}
$$


$B$ 's with $\Phi(B)=y^{k} \leq x^{k}, B \sim A$, and with $\Phi(B) \in \mathcal{A}$, because $\Phi(\mathcal{A})$ is a downset. Consequently

$$
|\mathcal{P}(\mathcal{A})|=2 \sum_{x^{k} \in W}\left|x^{k}\right|-2\left(\begin{array}{c}
k+1 \\
2
\end{array}\right)|\mathcal{A}|=2|| W||-2 \alpha\left(\begin{array}{c}
k+1 \\
2
\end{array}\right) .
$$

Thus our theorem follows from Lemma 1 and (1.3).

From now on we study our problem in the "sum-rank" version.

\section{From the Discrete to a Continuous Model}

A natural idea to solve a discrete problem for "good parameters" is to study the related continuous problem. Every $z^{k} \in \mathbb{Z}^{k}$ we let correspond to a cube $C\left(z^{k}\right) \triangleq\left\{x^{k}:\left\lceil x_{i}\right\rceil=z_{i}\right\}$ in $\mathbb{R}^{k}$. This mapping sends our $S_{U, k}$ for $U \in \mathbb{Z}^{+}$to $\stackrel{\sim}{\rightarrow} S_{U, k} \triangleq\left\{x^{k}: 0<x_{1}<x_{2} \cdots<x_{k} \leq U,\left\lceil x_{i}\right\rceil \neq\left\lceil x_{j}\right\rceil\right.$, if $\left.i \neq j\right\}$. Thus, keeping the partial order " $\leq$ ", we can "embed" our $\mathcal{L}(U, k)$ into a "continuous lattice" $\stackrel{\sim}{\rightarrow} \mathcal{L}(U, k)=\left(\stackrel{\sim}{\rightarrow} S_{U, k}, \leq\right)$. Moreover, the image $\stackrel{\sim}{\rightarrow} W \triangleq \Phi(W)$ of a downset $W$ in $\mathcal{L}(U, k)$ is a downset in $\stackrel{\sim}{\rightarrow} \mathcal{L}(U, k)$, with (finite) integer-components for maximal points. Let $\mu$ be the Lebesgue measure on $\mathbb{R}^{k^{\prime}}$, and let $k^{\prime} \leq k$ be specified by the context. For $W \subset \mathbb{R}^{k}$, define

$$
\|W\|=\int_{W}\left|x^{k}\right| d \mu, \text { where }\left|x^{k}\right|=\sum_{j} x_{j} .
$$

Let $\mathcal{D}$ be the set of downsets in $\stackrel{\sim}{\rightarrow} \mathcal{L}(U, k)$ with finitely many maximal points. Since it is of no consequence if we add or substract a set of measure zero, we will frequently exchange " $<$ " (or " $>$ ") and " $\leq$ " (or " $\geq$ ") in the sequel. It is enough in our problem for "good $\alpha$ " to consider $\max _{\mu(\stackrel{\sim}{\rightarrow} W)=\alpha, \stackrel{\sim}{\rightarrow} W \in \mathcal{D}}\|W\|$ in $\stackrel{\sim}{\rightarrow} \mathcal{L}(U, k)$, and the following lemma is the desired bridge.

Lemma 2. Suppose that $\stackrel{\sim}{\rightarrow} W \in \mathcal{D}$ has only maximal points with integer components, and so for a $W \subset \mathcal{L}(U, k) \stackrel{\sim}{\rightarrow} W=\Phi(W)$.

Then

$$
\|\stackrel{\sim}{\rightarrow} W\|=\|W\|-\frac{k}{2} \alpha, \quad \text { where } \alpha=\mu(\stackrel{\sim}{\rightarrow} W) .
$$

Proof.

$$
\begin{aligned}
&\|\stackrel{\sim}{\rightarrow} W\|=\sum_{z^{k} \in W}\left\|C\left(z^{k}\right)\right\|=\sum_{z^{k} \in W} \int_{C\left(z^{k}\right)}\left|x^{k}\right| \mu\left(d x^{k}\right) \\
&=\sum_{z^{k} \in W} \int_{z_{k}-1}^{z_{k}} d x_{k} \ldots \int_{z_{1}-1}^{z_{1}} d x_{1} \sum_{j=1}^{k} x_{j} \\
&=\sum_{z^{k} \in W} \sum_{i=1}^{k} \int_{z_{i}-1}^{z_{i}} x_{i} d x_{i}=\sum_{z^{k} \in W} \sum_{i=1}^{k} \frac{1}{2}\left(2 z_{i}-1\right)
\end{aligned}
$$

and (3.2) follows, because $|W|=\mu(\stackrel{\sim}{\rightarrow} W)$. We say that $W \in \mathcal{D}$ can be reduced to $W^{\prime} \in \mathcal{D}$, if $\mu\left(W^{\prime}\right)=\mu(W)$ and $\left\|W^{\prime}\right\| \geq\|W\|$. 


\section{Cones and Trapezoids}

Next we define cones and trapezoids, which will play important role in our problem. A cone in $\stackrel{\sim}{\rightarrow} S_{U, k}$ is a set

$$
K_{k}(u)=\left\{x^{k} \in R^{k}: 0<x_{1}<\cdots<x_{k} \leq u \text { and }\left\lceil x_{i}\right\rceil \neq\left\lceil x_{j}\right\rceil \text { for } i \neq j\right\} \text {, with } u \leq U .
$$

Clearly, $\stackrel{\sim}{\rightarrow} S_{U, k}$ is a cone itself. It can be denoted by $K_{k}(U)$. A trapezoid $R_{k}(v, u)$ in $K_{k}(U)$ is a downset below $(v, u \ldots u)$, where $0<v \leq u \leq U$, i.e.

$$
R_{k}(v, u) \triangleq\left\{x^{k} \in \stackrel{\sim}{\rightarrow} S_{U, k}: x_{1} \leq v, x_{k} \leq u\right\}
$$

and therefore $K_{k}(u)=R_{k}(u, u)$. Moreover, for $W \subset K_{k}(u)$ set

$$
\bar{W}^{(u)} \triangleq K_{k}(u) \backslash W
$$

and

$$
\hat{W}^{(u)} \triangleq\left\{(\lfloor u\rfloor, \ldots,\lfloor u\rfloor)-x^{k}: x^{k} \in \bar{W}^{(u)}\right\} .
$$

For integral $u$ one can easily verify that

$$
W=\hat{V}^{(u)} \text { for } \quad V=\hat{W}^{(u)}
$$

and

$$
R_{k}(v, u)=\hat{K}_{k}^{(u)}(u-v)
$$

Lemma 3. For $W \in \mathcal{D}$ and $W \subset K_{k}(u), u \leq U$,

$$
\|W\|=\left\|K_{k}(u)\right\|-k\lfloor u\rfloor \mu\left(\hat{W}^{(u)}\right)+\left\|\hat{W}^{(u)}\right\| .
$$

Proof. According to the definitions of "^ $(u)$ " and " $\|$ " ,

$$
\begin{gathered}
\|W\|=\int_{W}\left|x^{k}\right| \mu\left(d x^{k}\right)=\int_{K_{k}(u) \backslash \bar{W}^{(u)}}\left|x^{k}\right| \mu\left(d x^{k}\right) \\
=\left\|K_{k}(u)\right\|-\int_{\bar{W}^{(u)}}\left|x^{k}\right| \mu\left(d x^{k}\right) \\
=\left\|K_{k}(u)\right\|-\int_{\hat{W}^{(u)}} \sum_{j=1}^{k}\left(\lfloor u\rfloor-x_{j}\right) \mu\left(d x^{k}\right) \\
=\left\|K_{k}(u)\right\|-k\lfloor u\rfloor \mu\left(\hat{W}^{(u)}\right)+\left\|\hat{W}^{(u)}\right\| .
\end{gathered}
$$

Notice that for $u \notin \mathbb{Z}^{+} \hat{W}^{(u)}$ is not in $\mathcal{L}(u, k)$.

Corollary 1. For $u \in \mathbb{Z}^{+}$

$$
\left\|K_{k}(u)\right\|=\frac{k u}{2} \mu\left(K_{k}(u)\right) .
$$

Proof. One can verify (4.8) by standard techniques in calculus for evaluating integrals, however, Lemma 3 provides a very elegant and simple way. 
By (4.7) for $W \subset K_{k}(u)$

$$
\|W\|-\left\|\hat{W}^{(u)}\right\|=\left\|K_{k}(u)\right\|-k u \mu\left(\hat{W}^{(u)}\right)
$$

and by (4.5) and (4.7) one can exchange the roles of $W$ and $\hat{W}$. Therefore we have

$$
\left\|\hat{W}^{(u)}\right\|-\|W\|=\left\|K_{k}(u)\right\|-k u \mu(W) .
$$

"Adding (4.9) and (4.10)" and using the fact $\mu\left(K_{k}(u)\right)=\mu(W)+\mu\left(\hat{W}^{(u)}\right)$, we obtain (4.8). Next we establish a connection between $\left\|K_{k}(u)\right\|$ and $\mu\left(K_{k}(u)\right)$ for not necessarily integral $u$. It can elegantly be expressed in terms of densities. We define the density of $W \subset \mathbb{R}^{k^{\prime}}\left(k^{\prime} \leq k\right.$ defined by context) as

$$
d_{k^{\prime}}(W)=\frac{\|W\|}{\mu(W)} \text { and set } d=d_{k} .
$$

Then Corollary 1 takes the form

$$
d\left(K_{k}(u)\right)=\frac{k}{2} u, u \in \mathbb{Z}^{+} .
$$

We extend this formula to general $u$.

Lemma 4. For $u \leq U$ not necessarily integers, denote by $\theta \triangleq\{u\}=u-\lfloor u\rfloor$ the fractional part of $u$. Then

$$
\begin{gathered}
\mu\left(K_{k}(u)\right)=\left(\begin{array}{c}
\lfloor u\rfloor \\
k
\end{array}\right)+\theta\left(\begin{array}{c}
\lfloor u\rfloor \\
k-1
\end{array}\right), \\
\left\|K_{k}(u)\right\|=\frac{k u}{2} \mu\left(K_{k}(u)\right)+\frac{k-1}{2} \theta(1-\theta)\left(\begin{array}{c}
\lfloor u\rfloor \\
k-1
\end{array}\right)
\end{gathered}
$$

and therefore

(iii)

$$
d\left(K_{k}(u)\right)=\frac{k u}{2}+\frac{\frac{k-1}{2} \theta(1-\theta)}{\frac{1}{k}(\lfloor u\rfloor+1-k)+(k-1) \theta} .
$$

Proof. By its definition

$$
\begin{gathered}
K_{k}(u)=K_{k}(\lfloor u\rfloor) \cup\left\{x^{k}:\lfloor u\rfloor\right. \\
\left.<x_{k} \leq u \text { and }\left(x_{1}, \ldots, x_{k-1}\right) \in K_{k-1}(\lfloor u\rfloor)\right\} \\
\triangleq K_{k}(\lfloor u\rfloor) \cup J \text { (say). }
\end{gathered}
$$

On the other hand, according to the correspondence $\Phi$ between the discrete and the continuous models,

$$
\mu\left(K_{k}(\lfloor u\rfloor)\right)=\left(\begin{array}{c}
\lfloor u\rfloor \\
k
\end{array}\right), \mu\left(K_{k-1}(\lfloor u\rfloor)\right)=\left(\begin{array}{c}
\lfloor u\rfloor \\
k-1
\end{array}\right) .
$$

Therefore $\mu(J)=\theta\left(\begin{array}{c}\lfloor u\rfloor \\ k-1\end{array}\right)$ and consequently (i) holds. Now

$$
\left\|K_{k}(u)\right\|=\left\|K_{k}(\lfloor u\rfloor)\right\|+\|J\| .
$$


By Corollary 1 and (4.14)

$$
\left\|K_{k}(\lfloor u\rfloor)\right\|=\frac{k\lfloor u\rfloor}{2}\left(\begin{array}{c}
\lfloor u\rfloor \\
k
\end{array}\right) .
$$

Furthermore, by (4.8) for $k-1$ and by (4.14)

$$
\begin{gathered}
\|J\|=\mu\left(K_{k-1}(\lfloor u\rfloor) \int_{\lfloor u\rfloor}^{u} x_{k} d x_{k}+\int_{\lfloor u\rfloor}^{u} d x_{k}\left\|K_{k-1}(\lfloor u\rfloor)\right\|\right. \\
=\left(\lfloor u\rfloor+\frac{\theta}{2}\right) \theta\left(\begin{array}{c}
\lfloor u\rfloor \\
k-1
\end{array}\right)+\theta \frac{k-1}{2}\lfloor u\rfloor\left(\begin{array}{c}
\lfloor u\rfloor \\
k-1
\end{array}\right) .
\end{gathered}
$$

Combination of these three identities gives

$$
\left\|K_{k}(u)\right\|=\frac{k\lfloor u\rfloor}{2}\left(\begin{array}{c}
\lfloor u\rfloor \\
k
\end{array}\right)+\left(\lfloor u\rfloor+\frac{\theta}{2}+\frac{k-1}{2}\lfloor u\rfloor\right) \theta\left(\begin{array}{c}
\lfloor u\rfloor \\
k-1
\end{array}\right)
$$

and thus

$$
\left\|K_{k}(u)\right\|=\frac{k\lfloor u\rfloor}{2}\left(\begin{array}{c}
\lfloor u\rfloor \\
k
\end{array}\right)+\left(\frac{k+1}{2}\lfloor u\rfloor+\frac{\theta}{2}\right) \theta\left(\begin{array}{c}
\lfloor u\rfloor \\
k-1
\end{array}\right) .
$$

This and (i) imply

$$
\begin{gathered}
\left\|K_{k}(u)\right\|-\frac{k u}{2} \mu\left(K_{k}(u)\right)=-\frac{k \theta}{2}\left(\begin{array}{c}
\lfloor u\rfloor \\
k
\end{array}\right)+\left(\frac{\lfloor u\rfloor}{2}-\frac{k-1}{2} \theta\right) \theta\left(\begin{array}{c}
\lfloor u\rfloor \\
k-1
\end{array}\right) \\
=-\frac{k \theta}{2}\left(\begin{array}{c}
\lfloor u\rfloor \\
k
\end{array}\right)+\frac{\lfloor u\rfloor}{2} \theta\left(\begin{array}{c}
\lfloor u\rfloor \\
k-1
\end{array}\right)-\frac{k-1}{2} \theta^{2}\left(\begin{array}{c}
\lfloor u\rfloor \\
k-1
\end{array}\right) \\
=-\frac{\theta\lfloor u\rfloor}{2}\left(\begin{array}{c}
\lfloor u\rfloor-1 \\
k-1
\end{array}\right)+\frac{\lfloor u\rfloor}{2} \theta\left(\begin{array}{c}
\lfloor u\rfloor \\
k-1
\end{array}\right)-\frac{k-1}{2} \theta^{2}\left(\begin{array}{c}
\lfloor u\rfloor \\
k-1
\end{array}\right) \\
=\frac{\lfloor u\rfloor}{2} \theta\left(\begin{array}{c}
\lfloor u\rfloor-1 \\
k-2
\end{array}\right)-\frac{k-1}{2} \theta^{2}\left(\begin{array}{c}
\lfloor u\rfloor \\
k-1
\end{array}\right)=\frac{k-1}{2} \theta\left(\begin{array}{c}
\lfloor u\rfloor \\
k-1
\end{array}\right)-\frac{k-1}{2} \theta^{2}\left(\begin{array}{c}
\lfloor u\rfloor \\
k-1
\end{array}\right),
\end{gathered}
$$

and therefore (ii).

\section{Remark 1 (to Lemma 4).}

Actually, we can derive a somewhat more general result along the same lines. Let $J_{k}\left(u, u^{\prime}\right) \triangleq\left\{\left(x_{1}, \ldots, x_{k}\right) \mid u<x_{1}<\cdots<x_{k} \leq u^{\prime}\right.$ and $\left\lceil x_{i}\right\rceil \neq\left\lceil x_{j}\right\rceil$, for $i \neq j\}, u<u^{\prime} \in \mathbb{R}, \theta \triangleq\lceil u\rceil-u$ and $\theta^{\prime}=u^{\prime}-\left\lfloor u^{\prime}\right\rfloor \triangleq\left\{u^{\prime}\right\}$, then

$$
\mu\left(J_{k}\left(u, u^{\prime}\right)\right)=\left(\begin{array}{c}
\left\lfloor u^{\prime}\right\rfloor-\lceil u\rceil \\
k
\end{array}\right)+\left(\begin{array}{c}
\left\lfloor u^{\prime}\right\rfloor-\lceil u\rceil \\
k-1
\end{array}\right)\left(\theta+\theta^{\prime}\right)+\theta \theta^{\prime}\left(\begin{array}{c}
\left\lfloor u^{\prime}\right\rfloor-\lceil u\rceil \\
k-2
\end{array}\right)
$$

and

$\left\|J_{k}\left(u, u^{\prime}\right)\right\|-k\left(u+u^{\prime}\right)=\frac{k-1}{2}\left[\left(\theta^{\prime}-\theta\right)\left[1-\left(\theta+\theta^{\prime}\right)\right]\right]\left(\begin{array}{c}\left\lfloor u^{\prime}\right\rfloor-\lceil u\rceil \\ k-1\end{array}\right)-\frac{\theta \theta^{\prime}}{2}\left(\theta^{\prime}-\theta\right)\left(\begin{array}{c}\left\lfloor u^{\prime}\right\rfloor-\lceil u\rceil \\ k-2\end{array}\right)$.

This can be seen as follows.

By shifting the origin, we can assume w.l.o.g., that $u=-\theta, \theta \in[0,1)$, i.e. $\lfloor u\rfloor=0$. Then

$$
\begin{gathered}
J_{k}\left(u, u^{\prime}\right)=K_{k}\left(\left\lfloor u^{\prime}\right\rfloor\right) \cup\left(\left\{x_{1}:-\theta<x_{1} \leq 0\right\} \times\left\{\left(x_{2}, \ldots, x_{k}\right):\left(x_{2}, \ldots, x_{k}\right) \in K_{k-1}\left(\left\lfloor u^{\prime}\right\rfloor\right)\right)\right. \\
\cup\left(\left\{\left(x_{1}, \ldots, x_{k-1}\right):\left(x_{1}, \ldots, x_{k-1}\right) \in K_{k-1}\left(\left\lfloor u^{\prime}\right\rfloor\right)\right\} \times\left\{x_{k}:\left\lfloor u^{\prime}\right\rfloor<x_{k} \leq u^{\prime}\right\}\right) \\
\cup\left(\left\{x_{1}:-\theta<x_{1} \leq 0\right\} \times\left\{\left(x_{2}, \ldots, x_{k-1}\right) \in K_{k-2}\left(\left\lfloor u^{\prime}\right\rfloor\right)\right\} \times\left\{x_{k}:\left\lfloor u^{\prime}\right\rfloor<x_{k} \leq u^{\prime}\right\}\right)
\end{gathered}
$$

and by the same argument as the one used in the proof of Lemma 4 we obtain (4.19) and (4.20). 


\section{The Cases $k=2,3$}

Using the same idea as in the proof of Theorem 1 in [2] simple calculations lead to two alternatives.

Lemma 5. For $k=2, U \in \mathbb{Z}^{+}$and $W \in \mathcal{D}$ consider

$$
m_{1}(W) \triangleq \max \{x:(x, y) \in W \text { for some } y\} .
$$

Then

(i) $W$ can be reduced to a trapezoid, if $m_{1}(W) \leq \frac{U}{2}$ and

(ii) $W$ can be reduced to a cone, if $m_{1}(W) \geq \frac{U}{2}$.

Now we turn our attention to $k=3$ and drop all subscripts $k$ (for example write $K(U)$ instead of $K_{3}(U)$ and so on).

For $W \subset K(U)$ we call the 2-dimensional set

$$
S_{u}(W) \triangleq\{(x, y):(x, y, u) \in W \text { and }(x, y, u+\varepsilon) \notin W \text { for all } \varepsilon>0\}
$$

a $Z$-surface of $W$ at $u$.

We call this surface regular, when for some $(x, y) \in S_{u}(W)$ and some $\varepsilon>0$ $(x, y, u+\varepsilon) \in K(U)$. Therefore $S_{u}(W)$ is irregular iff $u=U$. The $Y-$ and $X$-surfaces are defined analogously. We present now the basic idea of "moving top layers from lower density to higher density".

Observe first that the condition $\mu(R(\nu, u))=\alpha$ (for fixed $\alpha$ ) forces $v$ to depend continuously on $u$, say

$$
v=V_{\alpha}(u)
$$

There are again two alternatives.

Lemma 6. For $k=3, u \leq U$, and $U \in \mathbb{Z}^{+}$any trapezoid $R(v, u)$ can be reduced to a cone or the trapezoid $R\left(V_{\alpha}(U), U\right)$.

Proof. Fix $\alpha$ and $U \in \mathbb{Z}^{+}$. Then $\left\|R\left(V_{\alpha}(u), u\right)\right\|$ is a continuous function in $u$, which achieves a maximal value. So, if the lemma is not true, then there are a $U \in \mathbb{Z}^{+}$, an $\alpha$, and a $u_{0}$ with $v_{0} \triangleq V_{\alpha}\left(u_{0}\right)<u_{0}<U$ and $R\left(v_{0}, u_{0}\right)$ achieves the maximal value. $R\left(v_{0}, u_{0}\right)$ has one regular $Z$-surface and one regular $X$-surface, namely

$$
\begin{gathered}
S_{1} \triangleq\left\{(x, y): 0<x<y \leq\left\lceil u_{0}\right\rceil-1, x \leq v_{0} \text { and }\lceil x\rceil \neq\lceil y\rceil\right\} \\
\text { and } S_{2} \triangleq\left\{(y, z):\left\lceil v_{0}\right\rceil<y<z \leq u_{0} \text { and }\lceil y\rceil \neq\lceil z\rceil\right\} .
\end{gathered}
$$

$$
\text { (c.f. Figure 1) }
$$



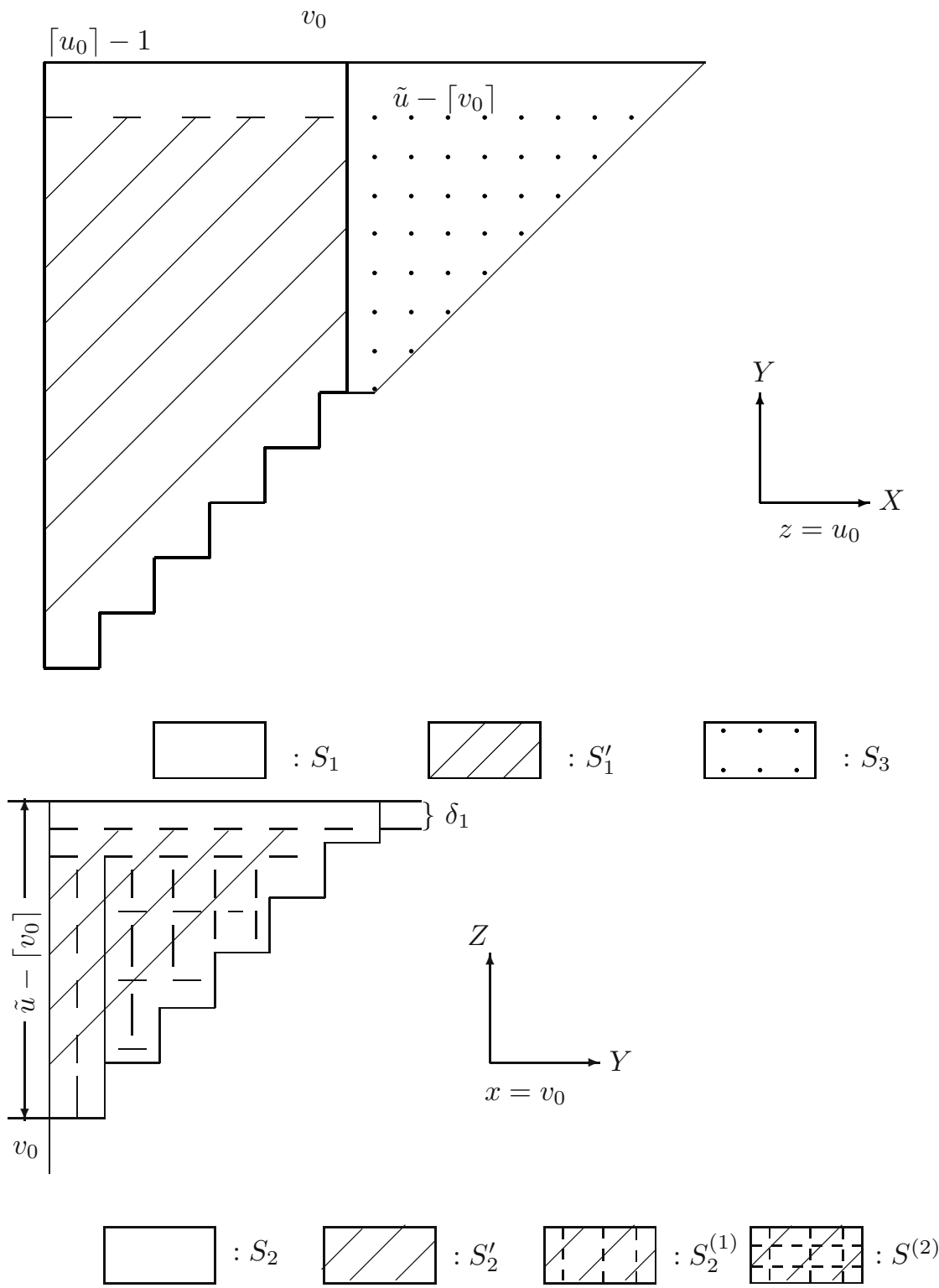

Fig. 1.

Case 1: $d\left(S_{1}\right)+u_{0}<d\left(S_{2}\right)+v_{0}$.

Choose $\delta_{1}, \delta_{2}>0$ and define

$$
\begin{gathered}
D_{1}=S_{1} \times\left\{z: u_{0}-\delta_{1}<z \leq u_{0}\right\} \\
\text { and } D_{2}=\left\{x: v_{0}<x \leq v_{0}+\delta_{2}\right\} \times S_{2}^{\prime} .
\end{gathered}
$$


They satisfy

$$
\begin{gathered}
\mu\left(D_{1}\right)=\mu\left(D_{2}\right), \\
\delta_{1} \leq u_{0}-\left(\left\lceil u_{0}\right\rceil-1\right), \delta_{2} \leq\left(\left\lfloor v_{0}\right\rfloor+1\right)-v_{0},
\end{gathered}
$$

and

$$
d\left(S_{1}\right)+u_{0}<d\left(S_{2}^{\prime \prime}\right)+v_{0} \leq d\left(S_{2}^{\prime}\right)+v_{0}
$$

where

$$
S_{2}^{\prime \prime} \triangleq S_{2} \backslash\left\{(y, z): u_{0}-\delta_{1}<z \leq u_{0}\right\}
$$

and

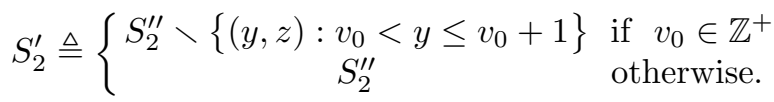

The second inequality in (5.12) follows from Lemma 4 and our choice is possible by (5.7). Then

$$
R^{\prime} \triangleq\left(R\left(v_{0}, u_{0}\right) \backslash D_{1}\right) \cup D_{2} \in \mathcal{D}
$$

is a trapezoid with measure $\alpha$.

However by (5.9) - (5.14),

$$
\begin{gathered}
\left\|R^{\prime}\right\|-\left\|R\left(v_{0}, u_{0}\right)\right\|=\left\|D_{2}\right\|-\left\|D_{1}\right\| \\
=\left[\mu\left(S_{2}^{\prime}\right) \int_{v_{0}}^{v_{0}+\delta_{2}} x d x+\delta_{2}\left\|S_{2}^{\prime}\right\|\right]-\left[\left\|S_{1}\right\| \delta_{1}+\mu\left(S_{1}\right) \int_{u_{0}-\delta_{1}}^{u_{0}} z d z\right] \\
=\left[\left(\mu\left(S_{2}^{\prime}\right) \delta_{2}\right)\left(v_{0}+\frac{\delta_{2}}{2}\right)+\left(\delta_{2} \mu\left(S_{2}^{\prime}\right)\right) d\left(S_{2}^{\prime}\right)\right]-\left[\left(\mu\left(S_{1}\right) \delta_{1}\right) d\left(S_{1}\right)+\left(\mu\left(S_{1}\right) \delta_{1}\right)\left(u_{0}-\frac{\delta_{1}}{2}\right)\right] \\
=\mu\left(D_{2}\right)\left[v_{0}+\frac{\delta_{2}}{2}+d\left(S_{2}^{\prime}\right)\right]-\mu\left(D_{1}\right)\left[d\left(S_{1}\right)+u_{0}-\frac{\delta_{1}}{2}\right. \\
=\mu\left(D_{1}\right)\left[\left(d\left(S_{2}^{\prime}\right)+v_{0}\right)-\left(d\left(S_{1}\right)+u_{0}\right)+\frac{\delta_{1}+\delta_{2}}{2}\right]>0,
\end{gathered}
$$

a contradiction. Here the fourth equality follows from $\mu\left(S_{2}^{\prime}\right) \delta_{2}=\mu\left(D_{2}\right)$ and $\mu\left(S_{1}\right) \delta_{1}=\mu\left(D_{2}\right)$ (by (5.9)), the fifth equality follows from (5.10) and the inequality follows from (5.12).

Case 2: $d\left(S_{1}\right)+u_{0}>d\left(S_{2}\right)+v_{0}$. One can come to a contradiction just like in case 1.

Case 3: $d\left(S_{1}\right)+u_{0}=d\left(S_{2}\right)+v_{0}$.

$S_{2}$ is a "shifted cone". One can calculate $d\left(S_{2}\right)$ and conclude with (5.16)

$$
\left\lceil u_{0}\right\rceil-2>v_{0}
$$

Consequently the following two surfaces are not empty:

$$
\begin{aligned}
& S_{1}^{\prime} \triangleq\left\{(x, y): 0<x<y \leq\left\lceil u_{0}\right\rceil-2, x \leq v_{0} \text { and }\lceil x\rceil \neq\lceil y\rceil\right\} \\
& \text { and } S_{2}^{(1)} \triangleq\left\{(y, z):\left\lceil v_{0}\right\rceil<y<z \leq u_{0}-1 \text { and }\lceil y\rceil \neq\lceil z\rceil\right\} \\
& =S_{2} \backslash\left\{(y, z): u_{0}-1<z \leq u_{0}\right\} \text {. }
\end{aligned}
$$


(See Figure 1) Assume first that

$$
\mu\left(S_{1}^{\prime}\right) \geq \mu\left(S_{2}^{(1)}\right)
$$

Let

$$
\begin{aligned}
& D_{1} \quad \triangleq\left\{(x, y, z) \in R\left(v_{0}, u_{0}\right): u_{0}-1<z \leq u_{0}\right\} \\
& =S_{1} \times\left\{z:\left\lceil u_{0}\right\rceil-1<z \leq u_{0}\right\} \cup S_{1}^{\prime} \times\left\{z: u_{0}-1<z \leq\left\lceil u_{0}\right\rceil-1\right\} \\
& \triangleq D_{1}^{\prime} \cup D_{1}^{\prime \prime} \\
& D_{2} \quad \triangleq\left\{(x, y, z) \in S_{U}: v_{0}<x \leq x_{0}, z \leq u_{0}-1\right\} \\
& =\left\{x: v_{0}<x \leq\left\lceil v_{0}\right\rceil\right\} \times S_{2}^{(1)} \cup\left[\bigcup_{i \geq 2}\left(\left\{x:\left\lceil v_{0}\right\rceil+i-1<x \leq v^{(i)}\right\} \times S_{2}^{(i)}\right)\right] \text {, }
\end{aligned}
$$

where

$$
S_{2}^{(i)}=S_{2}^{(i-1)} \backslash\left\{(y, z):\left\lceil v_{0}\right\rceil+2-i<x \leq\left\lceil v_{0}\right\rceil+3-i\right\},
$$

the last $v^{(i)}$ equals $x_{0}$, for the other $i$ 's $v^{(i)}=\left\lceil v_{0}\right\rceil+i$, and finally $x_{0}$ is specified by

$$
\mu\left(D_{1}\right)=\mu\left(D_{2}\right), \text { if such an } x_{0} \text { exists. }
$$

Otherwise continue with Case 4. Introduce now

$$
R^{\prime}=\left(R\left(v_{0}, u_{0}\right) \backslash D_{1}\right) \cup D_{2} .
$$

$R^{\prime}$ is a trapezoid with measure $\alpha$. Now we have, with justifications given afterwards,

$$
\begin{gathered}
\left\|D_{1}\right\| \quad\left[\mu\left(S_{1}\right)\left(u_{0}-\frac{u_{0}-\left\lceil u_{0}\right\rceil+1}{2}\right)\left(u_{0}-\left\lceil u_{0}\right\rceil+1\right)+\left\|S_{1}\right\|\left(u_{0}-\left\lceil u_{0}\right\rceil+1\right)\right] \\
+\left[\mu\left(S_{1}^{\prime}\right)\left(\left\lceil u_{0}\right\rceil-\frac{\left\lceil u_{0}\right\rceil-u_{0}}{2}-1\right)\left(\left\lceil u_{0}\right\rceil-u_{0}\right)+\left\|S_{1}^{\prime}\right\|\left(\left\lceil u_{0}\right\rceil-u_{0}\right)\right] \\
=\mu\left(D_{1}^{\prime}\right)\left(d\left(S_{1}\right)+u_{0}-\frac{u_{0}-\left\lceil u_{0}\right\rceil+1}{2}\right)+\mu\left(D_{1}^{\prime \prime}\right)\left[d\left(S_{1}^{\prime}\right)+\left\lceil u_{0}\right\rceil-\frac{\left\lceil u_{0}\right\rceil-u_{0}}{2}-1\right] \\
=\left[\mu\left(D_{1}^{\prime}\right) d\left(S_{1}\right)+\mu\left(D_{1}^{\prime \prime}\right) d\left(S^{\prime}\right)\right]+\left(u_{0}-1\right)\left(\mu\left(D_{1}^{\prime}\right)+\mu\left(D_{1}^{\prime \prime}\right)\right) \\
+\frac{1}{2} \mu\left(D_{1}^{\prime}\right)\left(u_{0}-\left\lceil u_{0}\right\rceil+1\right)+\frac{1}{2}\left(\left\lceil u_{0}\right\rceil-u_{0}\right)\left(2 \mu\left(D_{1}^{\prime}\right)+\mu\left(D_{1}^{\prime \prime}\right)\right) \\
<\mu\left(D_{1}\right)\left(d\left(S_{1}\right)+u_{0}-1\right)+\frac{1}{2}\left(u_{0}-\left\lceil u_{0}\right\rceil+1\right) \mu\left(D_{1}^{\prime}\right)+\frac{1}{2}\left(\left\lceil u_{0}\right\rceil-u_{0}\right)\left(2 \mu\left(D_{1}^{\prime}\right)+\mu\left(D_{1}^{\prime \prime}\right)\right) \\
=\mu\left(D_{1}\right)\left(d\left(S_{1}\right)+u_{0}-1\right)+\frac{1}{2}\left[\frac{\mu^{2}\left(D_{1}^{\prime}\right)}{\mu\left(S_{1}\right)}+2 \frac{\mu\left(D_{1}^{\prime}\right) \mu\left(D_{1}^{\prime \prime}\right)}{\mu\left(S_{1}^{\prime}\right)}+\frac{\mu\left(D_{1}^{\prime \prime}\right)^{2}}{\mu\left(S_{1}^{\prime}\right)}\right] \\
<\left(d\left(S_{1}\right)+u_{0}-1+\frac{\mu\left(D_{1}\right)}{2 \mu\left(S_{1}^{\prime}\right)}\right) \mu\left(D_{1}\right) .
\end{gathered}
$$

Here the second and the fourth equality are obtained by

$$
\mu\left(D_{1}^{\prime}\right)=\mu\left(S_{1}\right)\left(u_{0}-\left\lceil u_{0}\right\rceil+1\right) \text { and } \mu\left(D_{1}^{\prime \prime}\right)=\mu\left(S_{1}^{\prime}\right)\left(\left\lceil u_{0}\right\rceil-u_{0}\right) .
$$

The first inequality follows from $d\left(S_{1}\right)>d\left(S_{1}^{\prime}\right)$ and $\mu\left(D_{1}\right)=\mu\left(D_{1}^{\prime}\right)+\mu\left(D_{1}^{\prime \prime}\right)$ and the second one follows from $\mu\left(S_{1}\right)>\mu\left(S_{1}^{\prime}\right)$. Similarly, since $d\left(S_{2}^{(1)}\right)<d\left(S_{1}^{(i)}\right)$ and $\mu\left(S_{2}^{(1)}\right)>d\left(S_{2}^{(i)}\right)$ for $i \geq 2$

$$
\left\|D_{2}\right\|>\left(d\left(S_{2}^{(1)}\right)+v_{0}+\frac{\mu\left(D_{2}\right)}{2 \mu\left(S_{2}^{(1)}\right)}\right) \mu\left(D_{2}\right) .
$$


Finally, as $S_{2}$ and $S_{2}^{(1)}$ are shifted cones, by (iii) in Lemma 4, (5.6), (5.16), and (5.19)

$$
d\left(S_{2}^{(1)}\right)+v_{0}>d\left(S_{2}\right)-1+v_{0}=d\left(S_{1}\right)+u_{0}-1
$$

So a contradiction $\left\|R^{\prime}\right\|-\left\|R\left(v_{0}, u_{0}\right)\right\|=\left\|D_{2}\right\|-\left\|D_{1}\right\|>0$ follows from (5.19), (5.23), and (5.25). Therefore (5.20) must be false, i.e.

$$
\mu\left(S_{1}^{\prime}\right)<\mu\left(S_{2}^{(1)}\right)
$$

Let now $\stackrel{\sim}{\rightarrow} u \triangleq\left\lceil u_{0}\right\rceil-2, S_{3} \triangleq K(\stackrel{\sim}{\rightarrow} u) \backslash S_{1}^{\prime}$ (c.f. Figure 1$), \xi=1-\left\{v_{0}\right\}$, and $\eta=u_{0}-\left(\left\lceil u_{0}\right\rceil-1\right)$, then by $(5.26)$

$$
\mu\left(S_{3}\right)-\mu\left(S_{1}^{\prime}\right)>\mu\left(S_{3}\right)-\mu\left(S_{2}^{(1)}\right)=\left(\stackrel{\sim}{\rightarrow} u-\left\lceil v_{0}\right\rceil\right)(\xi-\eta),
$$

and by (i) in Lemma 4

$$
\mu\left(S_{3}\right)=\frac{1}{2}\left[\left(\stackrel{\sim}{\rightarrow} u-\left\lceil v_{0}\right\rceil\right)^{2}-\left(\stackrel{\sim}{\rightarrow} u-\left\lceil v_{0}\right\rceil\right)+2 \xi\left(\stackrel{\sim}{\rightarrow} u-\left\lceil v_{0}\right\rceil\right)\right]=\frac{\stackrel{\sim}{\rightarrow} u-\left\lceil v_{0}\right\rceil}{2}\left(\stackrel{\sim}{\rightarrow} u-\left\lceil v_{0}\right\rceil 1+2 \xi .\right.
$$

However, by their definitions

$$
\mu\left(S_{1}^{\prime}\right)+\mu\left(S_{3}\right)=\mu(K(\stackrel{\sim}{\rightarrow} u))=\frac{1}{2}\left(\stackrel{\sim}{\rightarrow} u^{2}-\stackrel{\sim}{\rightarrow} u\right) .
$$

Adding (5.27) to (5.29) we obtain

$$
\mu\left(S_{3}\right)>\frac{1}{4}(\stackrel{\sim}{\rightarrow} u-1) \stackrel{\sim}{\rightarrow} u+\frac{1}{2}\left(\stackrel{\sim}{\rightarrow} u-\left\lceil v_{0}\right\rceil\right)(\xi-\eta) .
$$

(5.28) and (5.30) imply

$$
\left(\stackrel{\sim}{\rightarrow} u-\left\lceil v_{0}\right\rceil\right)\left(\stackrel{\sim}{\rightarrow} u-\left\lceil v_{0}\right\rceil-1+\xi+\eta\right)>\frac{\stackrel{\sim}{\rightarrow} u}{2}(\stackrel{\sim}{\rightarrow} u-1) .
$$

Simplifying (5.31), we obtain

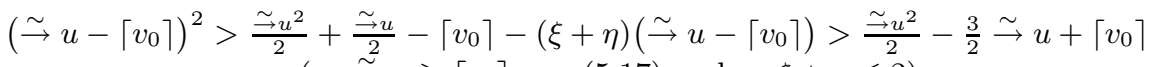

$$
\begin{aligned}
& \text { (as } \stackrel{\sim}{\rightarrow} u \geq\left\lceil v_{0}\right\rceil \text {, see }(5.17) \text { and as } \xi+\eta \leq 2 \text { ) } \\
& =\frac{1}{2}\left(\stackrel{\sim}{\rightarrow} u-\frac{3}{2}\right)^{2}-\frac{9}{8}+\left\lceil v_{0}\right\rceil \text {, i.e. } \\
& \stackrel{\sim}{\rightarrow} u-\left\lceil v_{0}\right\rceil>\frac{\sqrt{2}}{2} \stackrel{\sim}{\rightarrow} u-\frac{3 \sqrt{2}}{4}, \text { or } \\
& \left\lceil v_{0}\right\rceil<\left(1-\frac{\sqrt{2}}{2}\right) \stackrel{\sim}{\rightarrow} u+\frac{3 \sqrt{2}}{4}=\left(1-\frac{\sqrt{2}}{2}\right) \bar{u}-1+\frac{5 \sqrt{2}}{4},
\end{aligned}
$$

where $\bar{u} \triangleq\left\lceil u_{0}\right\rceil-1=\stackrel{\sim}{\rightarrow} u+1$. On the other hand, by (iii) in Lemma 4 and (5.16) with $\eta^{\prime}=\left\{u_{0}\right\}$

$$
\begin{gathered}
d\left(S_{1}\right)=d\left(S_{2}\right)+v_{0}-u_{0} \leq\left(u_{0}+\left\lceil v_{0}\right\rceil+\frac{\eta^{\prime}\left(1-\eta^{\prime}\right)}{\bar{u}-\left\lceil v_{0}\right\rceil-1}\right)+v_{0}-u_{0} \\
=v_{0}+\left\lceil v_{0}\right\rceil+\frac{\eta^{\prime}\left(1-\eta^{\prime}\right)}{\bar{u}-\left\lceil v_{0}\right\rceil-1}
\end{gathered}
$$


Consider that $S_{1}$ is the union of a rectangle and a 2-dimensional cone (a triangle).

$$
\begin{aligned}
\left\|S_{1}\right\|= & \frac{1}{2}\left(\left\lceil v_{0}\right\rceil^{2}-\left\lceil v_{0}\right\rceil\right)\left\lceil v_{0}\right\rceil+v_{0}\left(\bar{u}-\left\lceil v_{0}\right\rceil\right)\left(\left\lceil v_{0}\right\rceil+\frac{v_{0}+\bar{u}-\left\lceil v_{0}\right\rceil}{2}\right) \\
& =\frac{1}{2}\left[\left\lceil v_{0}\right\rceil^{2}\left(\left\lceil v_{0}\right\rceil-1\right)+v_{0}\left(\bar{u}-\left\lceil v_{0}\right\rceil\right)\left(v_{0}+\left\lceil v_{0}\right\rceil+\bar{u}\right)\right],
\end{aligned}
$$

and

$$
\mu\left(S_{1}\right)=\frac{1}{2}\left(\left\lceil v_{0}\right\rceil^{2}-\left\lceil v_{0}\right\rceil\right)+v_{0}\left(\bar{u}-\left\lceil v_{0}\right\rceil\right) .
$$

(5.33) - (5.35) imply

$$
\begin{gathered}
\left(v_{0}+\left\lceil v_{0}\right\rceil+\frac{\eta^{\prime}\left(1-\eta^{\prime}\right)}{\bar{u}-\left\lceil v_{0}\right\rceil-1}\right)\left(\frac{1}{2}\left(\left\lceil v_{0}\right\rceil^{2}-\left\lceil v_{0}\right\rceil\right)+v_{0}\left(\bar{u}-\left\lceil v_{0}\right\rceil\right)\right) \\
\geq \frac{1}{2}\left[\left\lceil v_{0}\right\rceil^{2}\left(\left\lceil v_{0}\right\rceil-1\right)+v_{0}\left(\bar{u}-\left\lceil v_{0}\right\rceil\right)\left(v_{0}+\left\lceil v_{0}\right\rceil+\bar{u}\right)\right], \text { i.e. } \\
\left\lceil v_{0}\right\rceil\left(\left\lceil v_{0}\right\rceil-1\right) \frac{\eta^{\prime}\left(1-\eta^{\prime}\right)}{\left.\bar{u}-\mid v_{0}\right\rceil-1} \geq v_{0}\left(\bar{u}-\left\lceil v_{0}\right\rceil\right)\left(\bar{u}-v_{0}-\left\lceil v_{0}\right\rceil-\frac{2 \eta^{\prime}\left(1-\eta^{\prime}\right)}{\bar{u}-\left\lceil v_{0}\right\rceil-1}\right)-v_{0}\left(\left\lceil v_{0}\right\rceil^{2}-\left\lceil v_{0}\right\rceil\right) \\
=v_{0}\left(\bar{u}^{2}-3\left\lceil v_{0}\right\rceil \bar{u}+\left\lceil v_{0}\right\rceil^{2}\right)+v_{0}\left\lceil v_{0}\right\rceil+v_{0}\left(\bar{u}-\left\lceil v_{0}\right\rceil\right)\left[\left(\left\lceil v_{0}\right\rceil-v_{0}\right)-\frac{2 \eta^{\prime}\left(1-\eta^{\prime}\right)}{\bar{u}-\left\lceil v_{0}\right\rceil-1}\right] \\
\geq\left(\left\lceil v_{0}\right\rceil-1\right)\left[\left(\bar{u}^{2}-3 \bar{u}\left\lceil v_{0}\right\rceil+\left\lceil v_{0}\right\rceil^{2}\right)+\left\lceil v_{0}\right\rceil-\left(\bar{u}-\left\lceil v_{0}\right\rceil\right) \frac{2 \eta^{\prime}\left(1-\eta^{\prime}\right)}{\bar{u}-\left\lceil v_{0}\right\rceil-1}\right],
\end{gathered}
$$

i.e.

$$
\begin{gathered}
\bar{u}^{2}-3 \bar{u}\left\lceil v_{0}\right\rceil+\left\lceil v_{0}\right\rceil^{2} \leq\left(2 \bar{u}-\left\lceil v_{0}\right\rceil\right) \frac{\eta^{\prime}\left(1-\eta^{\prime}\right)}{\bar{u}-\left\lceil v_{0}\right\rceil-1}-\left\lceil v_{0}\right\rceil \\
\leq \frac{1}{4} \frac{2 \bar{u}-\left\lceil v_{0}\right\rceil}{\bar{u}-\left\lceil v_{0}\right\rceil-1}-\left\lceil v_{0}\right\rceil .
\end{gathered}
$$

Comparing (5.32) and (5.36), one can conclude

$$
\begin{gathered}
{\left[\left(1-\frac{\sqrt{2}}{2}\right)+\frac{5 \sqrt{2}-4}{4 \bar{u}}\right]^{2}-3\left[\left(1-\frac{\sqrt{2}}{2}\right)+\frac{5 \sqrt{2}-4}{4 \bar{u}}\right]+1} \\
\quad<\frac{1}{\bar{u}} \cdot \frac{1}{2 \sqrt{2} \bar{u}-5 \sqrt{2}}-\frac{1}{\bar{u}}\left[\left(1-\frac{\sqrt{2}}{2}\right)+\frac{\sqrt{2}-4}{4 \bar{u}}\right] \\
=\frac{1}{\bar{u}}\left(\frac{1}{2 \sqrt{2} \bar{u}-5 \sqrt{2}}-\frac{5 \sqrt{2}-4}{4 \bar{u}}\right)-\frac{1}{\bar{u}}\left(1-\frac{\sqrt{2}}{2}\right), \text { or } \\
\left(1-\frac{\sqrt{2}}{2}\right)^{2}-3\left(1-\frac{\sqrt{2}}{2}\right)+1< \\
\frac{1}{4 \bar{u}}(3 \sqrt{2}+2)+\frac{1}{\bar{u}}\left(\frac{1}{2 \sqrt{2} \bar{u}-5 \sqrt{2}}-\frac{4(5 \sqrt{2}-4)+(5 \sqrt{2}-4)}{16 \bar{u}}\right) .
\end{gathered}
$$

One can check that (5.37) does not hold unless $\bar{u}<8$, or $\left\lceil u_{0}\right\rceil \leq 8$. However,

it is not difficult to check that (5.16) and (5.26) cannot hold simultaneously for $4<u \leq 8$. Finally using the condition $U \notin \mathbb{Z}^{+}$it follows that $U \geq 4$. One can also check the lemma for $3<u \leq 4$.

\section{Case 4}

If an $x_{0}$ with $\mu\left(D_{1}\right)=\mu\left(D_{2}\right)$ does not exist, i.e. $D_{1}$ is too big to find a $D_{2}$ with the same measure, we choose a proper $h, 0<h<1$, such that for

$$
\begin{gathered}
D_{1} \triangleq\left\{(x, y, z) \in R\left(v_{0}, u_{0}\right): u_{0}-h<z \leq u_{0}\right\} \text { and } \\
D_{2} \triangleq\left\{(x, y, z) \in S_{U}: v_{0}<x<y \leq u_{0}-h\right\}, \mu\left(D_{1}\right)=\mu\left(D_{2}\right) .
\end{gathered}
$$


$D_{2}$ is a shifted cone. By the arguments leading to Lemma 4, (c.f. (4.18), (4.19) in Remark to Lemma 4) we get for its density

$$
\begin{gathered}
d\left(D_{2}\right) \geq 3\left\lceil v_{0}\right\rceil+ \\
\frac{3}{2}\left[u_{0}-h-\left\lceil v_{0}\right\rceil-\left(1-\left\{v_{0}\right\}\right)\right]-\frac{\left\{v_{0}\right\}\left(1-\left\{v_{0}\right\}\right)}{\left|u_{0}-h-\left\lceil v_{0}\right\rceil\right|^{+}+2\left(1-\left\{v_{0}\right\}\right)} \\
=\frac{3}{2}\left(u_{0}+v_{0}-h\right)-\frac{\left\{v_{0}\right\}\left(1-\left\{v_{0}\right\}\right)}{\left|u_{0}-h-\left\lceil v_{0}\right\rceil\right|^{+}+2\left(1-\left\{v_{0}\right\}\right)} .
\end{gathered}
$$

However, by (5.16) and Lemma 4

$$
d\left(D_{1}\right)=d\left(S_{1}\right)+u_{0}-\frac{h}{2}=d\left(S_{2}\right)+v_{0}-\frac{h}{2} \leq v_{0}+\left\lceil v_{0}\right\rceil+u_{0}-\frac{h}{2}+\frac{1}{4} .
$$

Then

$$
\begin{aligned}
d\left(D_{2}\right)-d\left(D_{1}\right) \geq \frac{u_{0}}{2}+ & \frac{v_{0}}{2}-\left\lceil v_{0}\right\rceil-h-\frac{1}{4}-\frac{\left\{v_{0}\right\}\left(1-\left\{v_{0}\right\}\right)}{\left|u_{0}-h-\left\lceil v_{0}\right\rceil\right|^{+}+2\left(1-\left\{v_{0}\right\}\right)} \\
& >\frac{1}{2}\left(u_{0}-\left\lceil v_{0}\right\rceil\right)-h-\frac{3}{4} .
\end{aligned}
$$

Thus by (5.16), for $u_{0}>8$

$$
d\left(D_{2}\right)>d\left(D_{1}\right)
$$

For $\left\lceil u_{o}\right\rceil \leq 8$ we check it directly.

Remark 2. For $m \in \mathbb{Z}^{+}$denote by $\mathcal{D}_{m}$ the set of downsets of $\stackrel{\sim}{\rightarrow} \mathcal{L}(U)(\triangleq \stackrel{\sim}{\rightarrow}$ $\mathcal{L}(U, 3))$ with $m$ maximal points. We can show that $\max _{\mu(W)=\alpha, W \in \mathcal{D}_{m}}\|W\|$ can be achieved, as well.

More precisely, define a metric on the set $\left\{\left(x^{i}, y^{i}, z^{i}\right)_{i=1}^{k}:\left(x^{i}, y^{i}, z^{i}\right) \in \mathbb{R}^{3}\right\}$ as the sum of Euclidean (or $L_{1}-$ ) metrics of the $k$ components points. Then for fixed $\mu(W)=\alpha, W \in \mathcal{D}_{m},\|W\|$ is a continuous function of its maximal points.

\section{On Regular Surfaces}

Lemma 7. Every $W \in \mathcal{D}$ can be reduced to a $W^{\prime} \in \mathcal{D}$, which has of each of the regular $X-, Y-$ and $Z-$ surfaces at most one (for $U \in Z^{+}$).

Proof. Suppose there exists a $W$ that canot be reduced to such kind of $W^{\prime}$. W.l.o.g. by Remark 1 we assume $W$ achieves $\underset{m^{\prime} \leq m}{\longrightarrow} \max _{\mu}(W)=\alpha, W \in \mathcal{D}_{m^{\prime}}\|W\|$, (recalling $\mathcal{D}=\bigcup_{m=1}^{\infty} \mathcal{D}_{m}$ by its definition).

Case 1: Suppose $W$ has at least 2 regular $z$-surfaces, say $S_{i}$ at $i$, for $i=1,2$, and

$$
d\left(S_{1}\right)+u_{1} \leq d\left(S_{2}\right)+u_{2} .
$$


Using the same method as in the proof of Lemma 6, Case 1, one can obtain a contradiction. Furthermore, we can see that $W$ has 2 regular $X$-surfaces iff $\hat{W}^{(u)}$ has 2 regular $Z$-surfaces. Since $W$ and $\hat{W}^{(u)}$ must achieve the maximal value simultaneously, we are left with Case 2: $W$ has at least 2 regular $Y$-surfaces $S_{1}$ at $v_{1}$ and $S_{2}$ at $v_{2}$ with

$$
d\left(S_{1}\right)+v_{1} \leq d\left(S_{2}\right)+v_{2}
$$

and of each of the regular $Z$ - and $X$ - surfaces at most one. Let $S_{2}^{\prime}=S_{2}$, if $v_{2} \notin Z$, and otherwise let $S_{2}^{\prime}=S_{2} \backslash\left\{(x, z) \mid v_{1}<z \leq v_{1}+1\right\}$. Since $W$ has no 2 regular $Z$-surfaces nor $X$-surfaces, $S_{2}$ is rectangular, consequently $d\left(S_{2}^{\prime}\right)>d\left(S_{2}\right)$. Thus we can use $S_{2}^{\prime}$ to replace $S_{2}$ and play the same game as before to arrive at a contradiction.

\section{Main Result in Continuous Model, $k=3$}

Theorem 2. For $U \in \mathbb{Z}^{+}$and fixed $\alpha$ every $W \in \mathcal{D}$ with $\mu(W)=\alpha$ can be reduced to a cone or the trapezoid $R\left(V_{\alpha}(U), U\right)$.

Proof. Assume the theorem is not true. Then by Remark 1 and Lemma 6 there exists a $W \in \mathcal{D}$ with $m$ maximal points achieving maximal value of $\|W\|$ over $\bigcup_{m^{\prime} \leq m} \mathcal{D}_{m}$, which is neither a cone nor a trapezoid. Moreover, by Lemma 7 we can assume that $W$ has at most one regular $X-$, at most one regular $Y-$, and at most one regular $Z$ - surface.

Case 1: $W$ has only one (regular or irregular) $Z$-surface at $u \leq U$. Then $W$ has one or two maximal points, whose third components must be $u$. Subcase 1.1: $W$ has one maximal point, say $P=(w, v, u)$. Because $v=\lceil u\rceil-1$ implies $W$ is a trapezoid, we assume $w<v \leq\lceil u\rceil-1$. Thus, $W$ has one $Z$-surface $S_{1}$ and one $Y$-surface, which are shown in Figure 2 (a).

We are going to use the same idea as before. However, it is not enough to exchange the layers. Instead of it we will exchange cylinders. (a) Suppose $w \geq$ $u-\lceil v\rceil$.

We choose $0<h_{1}<u-\lceil v\rceil$ and define $S_{2} \triangleq\left\{(y, z): v<y<z \leq u-h_{1}\right.$ and $\lceil y\rceil \neq\lceil z\rceil\}, D_{1}=S_{1} \times\left\{z: u-h_{1}<z \leq u\right\}, D_{2} \triangleq\{x: 0<x \leq w\} \times S_{2}$, and $W^{\prime}=\left(W \backslash D_{1}\right) \cup D_{2}$ such that

$$
\mu\left(D_{1}\right)=\mu\left(D_{2}\right)
$$

Then $W^{\prime} \in \mathcal{D}$ and furthermore, if we denote $\{v\}$ by $\theta$ and use the arguments of the proof of Lemma 4 (see Remark to Lemma 4), then we obtain

$d\left(S_{2}\right)-\left(v+u-h_{1}\right)=\frac{\left(\theta^{\prime}-\bar{\theta}\right)\left[1-\left(\theta^{\prime}+\bar{\theta}\right)\right]-\bar{\theta} \theta^{\prime}\left(\theta^{\prime}-\bar{\theta}\right)\left(\left\lfloor u-h_{1}\right\rfloor-\lceil v\rceil\right)^{-1}}{\left(\left\lfloor u-h_{1}\right\rfloor-\lceil v\rceil-1\right)+2\left(\theta^{\prime}+\bar{\theta}\right)+2 \bar{\theta} \theta^{\prime}\left(\left\lfloor u-h_{1}\right\rfloor-\lceil v\rceil\right)^{-1}} \triangleq \eta_{1}$, 


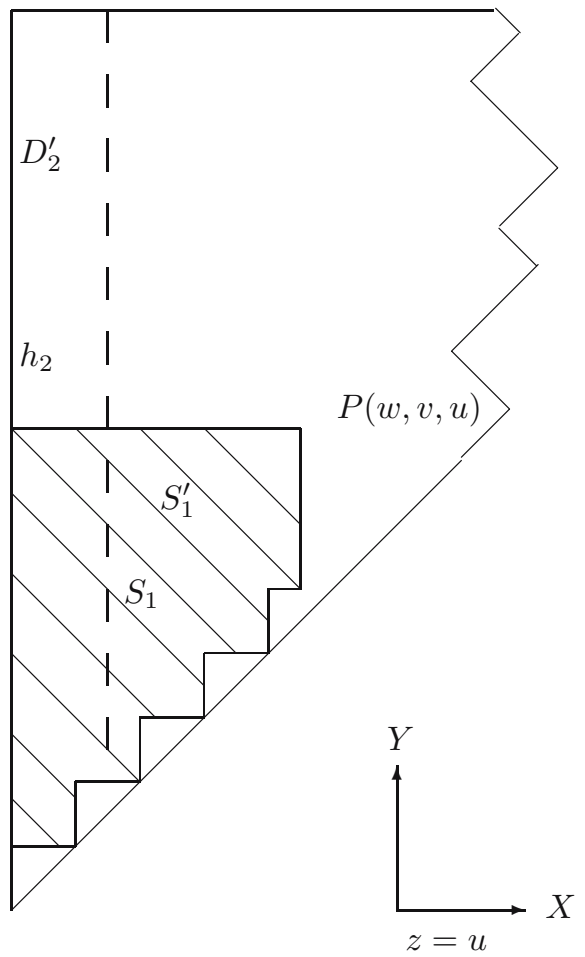

(a)
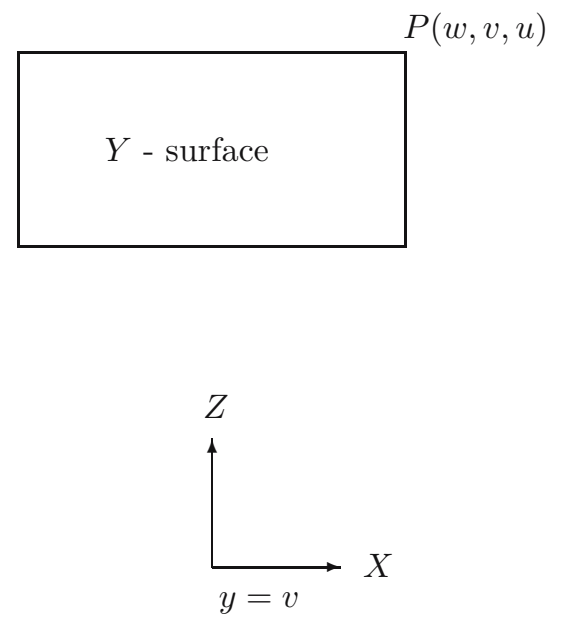

(b)

Fig. 2 (a).

where $\theta^{\prime} \triangleq\left\{u-h_{1}\right\}$ and $\bar{\theta}=1-\theta=\lceil v\rceil-v$, if $u-h_{1}-\lceil v\rceil>1$. By Lemma 4 and Corollary 2,

$$
d\left(S_{1}\right)-v \leq \frac{\theta(1-\theta)}{\lceil v\rceil-1+2 \theta} \triangleq \eta_{2}
$$

Consequently

$$
d\left(S_{2}\right)-\left(d\left(S_{1}\right)+u\right) \geq-h_{1}+\eta_{1}-\eta_{2} .
$$

Therefore, by simple calculation

$$
\begin{gathered}
\left\|W^{\prime}\right\|-\|W\|=\left\|D_{2}\right\|-\left\|D_{1}\right\| \\
=\mu\left(D_{2}\right)\left(d\left(S_{2}\right)+\frac{w}{2}\right)-\mu\left(D_{1}\right)\left(d\left(S_{1}\right)+u-\frac{h_{1}}{2}\right) \\
=\mu\left(D_{2}\right)\left[d\left(S_{2}\right)-\left(d\left(S_{1}\right)+u\right)+\frac{w}{2}+\frac{h_{1}}{2}\right] \geq \mu\left(D_{2}\right)\left[\frac{w}{2}-\frac{h_{1}}{2}+\eta_{1}-\eta_{2}\right] .
\end{gathered}
$$

By (7.2),

$$
\eta_{1} \geq-\frac{\bar{\theta}(1-\bar{\theta})}{\left\lfloor u-h_{1}\right\rfloor-\lceil v\rceil-1+2 \bar{\theta}}=\frac{-\theta(1-\theta)}{\left\lfloor u-h_{1}\right\rfloor-\lceil v\rceil-1+2(1-\theta)} .
$$




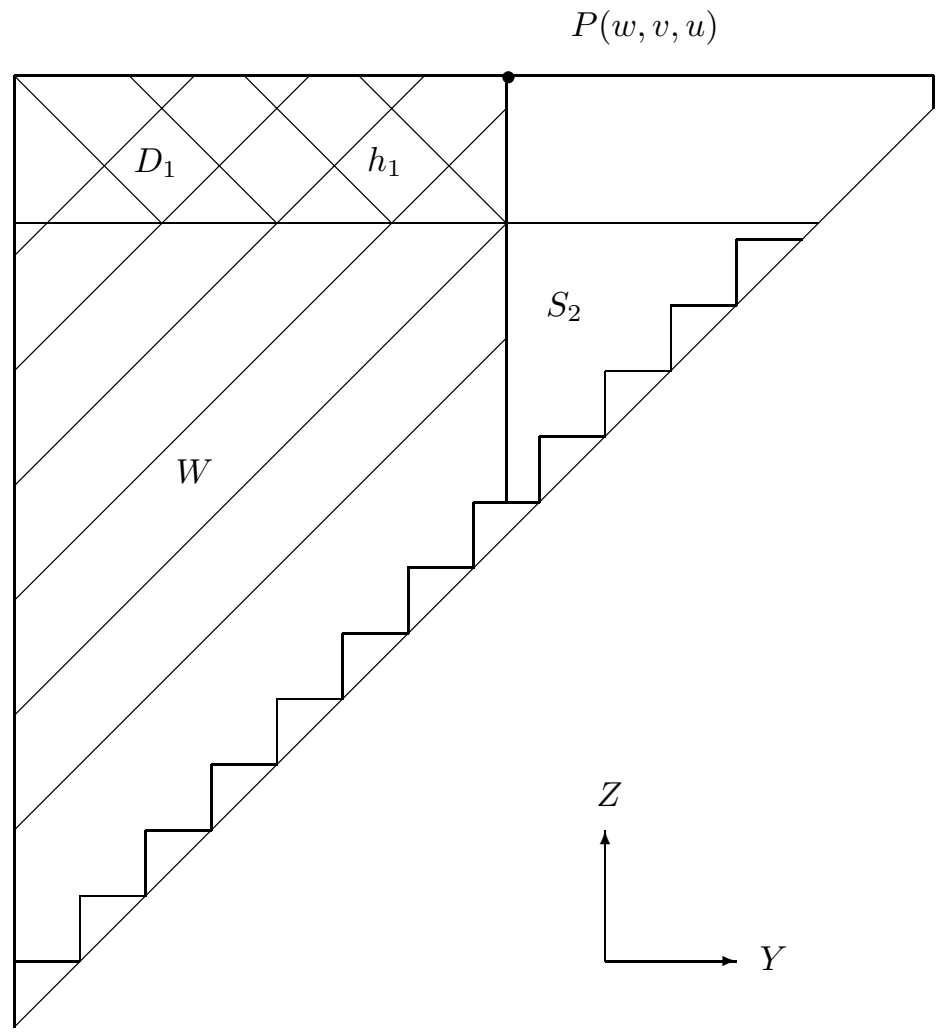

(c)

Fig. 2 (b).

Thus, (7.3) and (7.6) imply

$$
\eta_{1}-\eta_{2} \geq-\frac{1}{2}
$$

However, when $h_{1} \leq u-\lceil v\rceil-1,(7.5)$ and (7.2) imply the contradiction

$$
\left\|W^{\prime}\right\|>\|W\| .
$$

When $u-\lceil v\rceil-1 \leq h_{1}<u-\lceil v\rceil, S_{2}$ becomes a rectangle (c.f. Figure 3) and $d\left(S_{2}\right)=v+u-h_{1}+\frac{\bar{\theta}}{2}-\frac{u-\lceil v\rceil-h_{1}}{2}$. Then use

$$
\eta_{1}=\frac{1-\theta}{2}-\frac{u-\lceil v\rceil-h_{1}}{2},
$$

and (7.8) holds again. (b) If $w<u-\lceil v\rceil$, then we choose $0<h_{2}<w$ and let $S_{1}^{\prime}=S_{1} \backslash\left\{(x, y): 0<x \leq h_{2}\right\}, S_{2}^{\prime}=\{(y, z): v \leq y<z<u,\lceil y\rceil \neq\lceil z\rceil\}$, $D_{1}^{\prime} \triangleq S_{1}^{\prime} \times\{z:\lceil v\rceil<z \leq u\}$, and $D_{2}^{\prime}=S_{2} \times\left\{x: 0<x \leq h_{2}\right\}$ with 


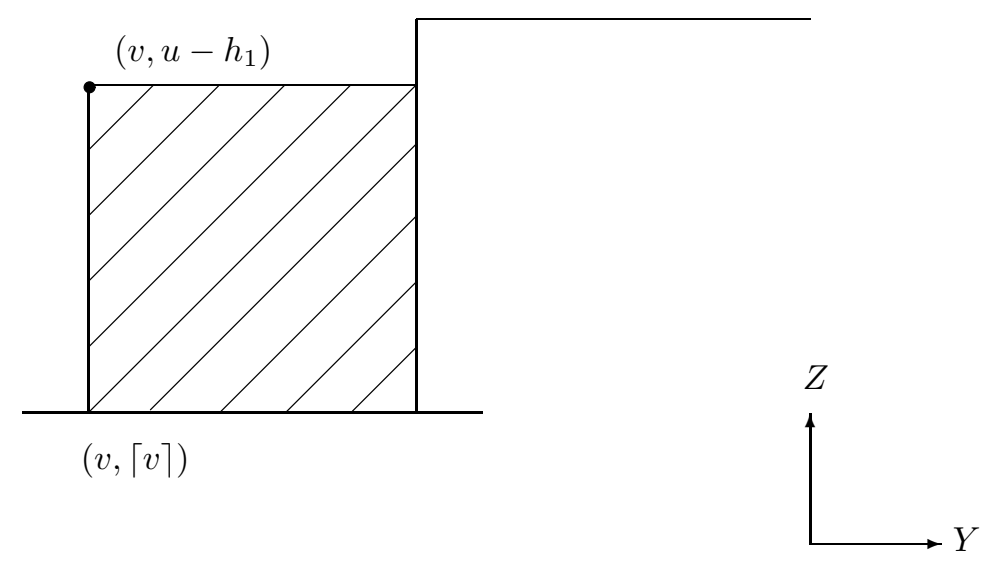

Fig. 3.

$\mu\left(D_{1}^{\prime}\right)=\mu\left(D_{2}^{\prime}\right)$. Considering $\left(W \backslash D_{1}^{\prime}\right) \cup D_{2}^{\prime}$ in a similar way we arrive at a contradiction. (c.f. Figure 2 (a)) Subcase 1.2: $W$ has 2 maximal points.

According to our assumption on regular surfaces the $Z$-surface $S_{1}$ of $W$ must be as in Figure 4.

Then we follow the same reasoning as in the previous subcase in the shadow part (i.e. exchange cylinders in the shadow part $\left\{(x, y, z) \in S_{U} \mid x \leq v_{o}\right\}$, where $v_{0}$ is the smaller first component in the 2 maximal points) and obtain a contradiction.

Case 2: $W$ has $2 Z$-surfaces. Since $W$ and $\hat{W}$ always simultaneously achieve their maximum, we can assume $\hat{W}$ has $2 \mathcal{Z}$-surfaces too, because otherwise we can use $\hat{W}$, which has been studied in Case 1 already, instead of $W$. However, $\hat{W}$ has $2 Z$-surfaces iff $W$ has one regular $X$-surface, and

$$
\left\{(0, y, z) \in S_{U}\right\} \backslash W \neq \varnothing .
$$

Thus we can assume $W$ has one regular $X$-surface and (7.10) holds.

Then by our assumption $W$ has 2 maximal points, say $P_{1}=\left(w_{1}, v_{1}, U\right)$ and $P_{2}=\left(w_{2}, v_{2}, u\right)$ and $v_{1}<\lceil U\rceil-1$. Subcase 2.1: $\left\lceil v_{1}\right\rceil \geq\lfloor u\rfloor$. Then $w_{1}<w_{2}$,

because $P_{2}$ is maximal. Recalling that in our proof under subcase 1.1 we only exchange the points $(x, y, z)$ with $x \leq w$, and $y \geq\lceil v\rceil$, in the present case we can use the plane $x=w_{1}$ to cut $S_{U}$ into 2 parts and repeat the same reasoning as in subcase 1.1 to obtain a contradiction in the part $x \geq w_{1}$.

Moreover, for this kind of $W$ 's, $\hat{W}^{(U)}$ has 2 maximal points, $\hat{P}_{1}=\left(\hat{w}_{1}, \hat{v}_{1}, U\right)$ and $\hat{P}_{2}=\left(\hat{w}_{2}, \hat{v}_{2}, \hat{u}\right)$ with $\hat{w}_{1}=U-\left\lceil v_{1}\right\rceil, \hat{v}_{1}=U-v_{1}, \hat{w}_{2}=U-u, \hat{v}_{2}=U-\left\lceil w_{1}\right\rceil$, $\hat{u}=U-w_{1}$, i.e. $\hat{w}_{1}=\left\lceil\hat{v}_{1}\right\rceil-1, \hat{v}_{2}=\lceil\hat{u}\rceil-1$ and $\hat{w}_{2} \geq \hat{w}_{1}$. Therefore, the following subcase 2.2 can be cancelled from our list. Subcase 2.2: $w_{1}=\left\lceil v_{1}\right\rceil-1$, 


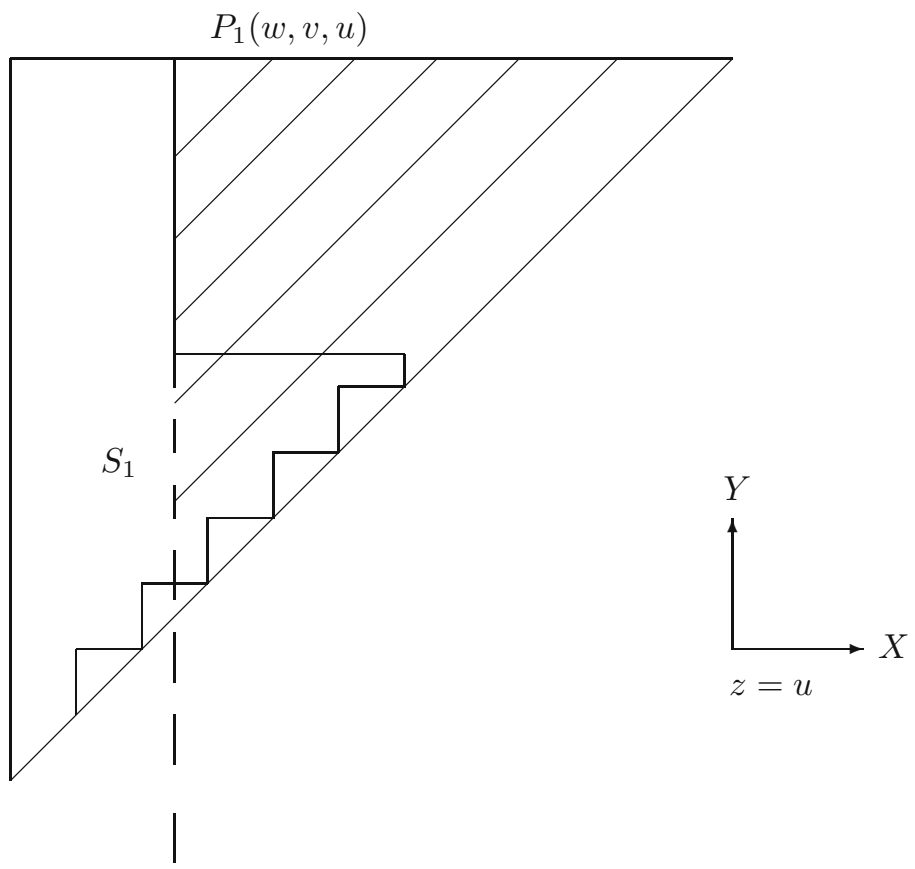

Fig. 4.

$v_{2}=\lceil u\rceil-1$, and $w_{2} \geq w_{1}$. Subcase 2.3: $w_{1}=\left\lceil v_{1}\right\rceil-1, v_{2}=\lceil u\rceil-1, w_{2}<w_{1}$, and $\left\lceil v_{1}\right\rceil<u$. In this subcase, there are one regular $Z$-surface and one regular $Y$-surface passing $P_{1}$.

Denote by $S_{1}=\left\{(x, y): y \leq v_{1}\lceil x\rceil \neq\lceil y\rceil\right\}$ the irregular $Z$-surface, by $S_{2}$ the regular $X$-surface at $w_{2}$, a shifted cone, and by $S_{3}=\{(y, z):\lceil y\rceil \neq\lceil z\rceil$, $\left.(0, y, z) \in S_{U} \backslash W\right\}$ as in Figure 5 .

Then $\stackrel{\sim}{\rightarrow} W \triangleq W \cap\left\{(x, y, z): y>v_{1}\right\}$ is a cylinder with base $S_{2}$. Therefore we can assume

$$
v_{2}-v_{1}=\lceil u\rceil-1-v_{1}>U-u,
$$

because otherwise, by Lemma 5 , we can replace $\stackrel{\sim}{\rightarrow} W$ by a cylinder with the same size 2-dimensional trapezoid base and the same height, and then reduce $W$ to a downset with 2 regular $Y$-surfaces. If $d\left(S_{1}\right)+U<d\left(S_{3}\right)$, then we can repeat our reasoning as before and arrive at a contradiction. So we only need to consider

$$
d\left(S_{1}\right)+U \geq d\left(S_{3}\right),
$$

which, in fact, is also impossible. By Lemma 4

$$
d\left(S_{1}\right)=v_{1}+\frac{\theta(1-\theta)}{\left|\left\lfloor v_{1}\right\rfloor-1\right|^{+}+2 \theta} \triangleq v_{1}+\eta .
$$




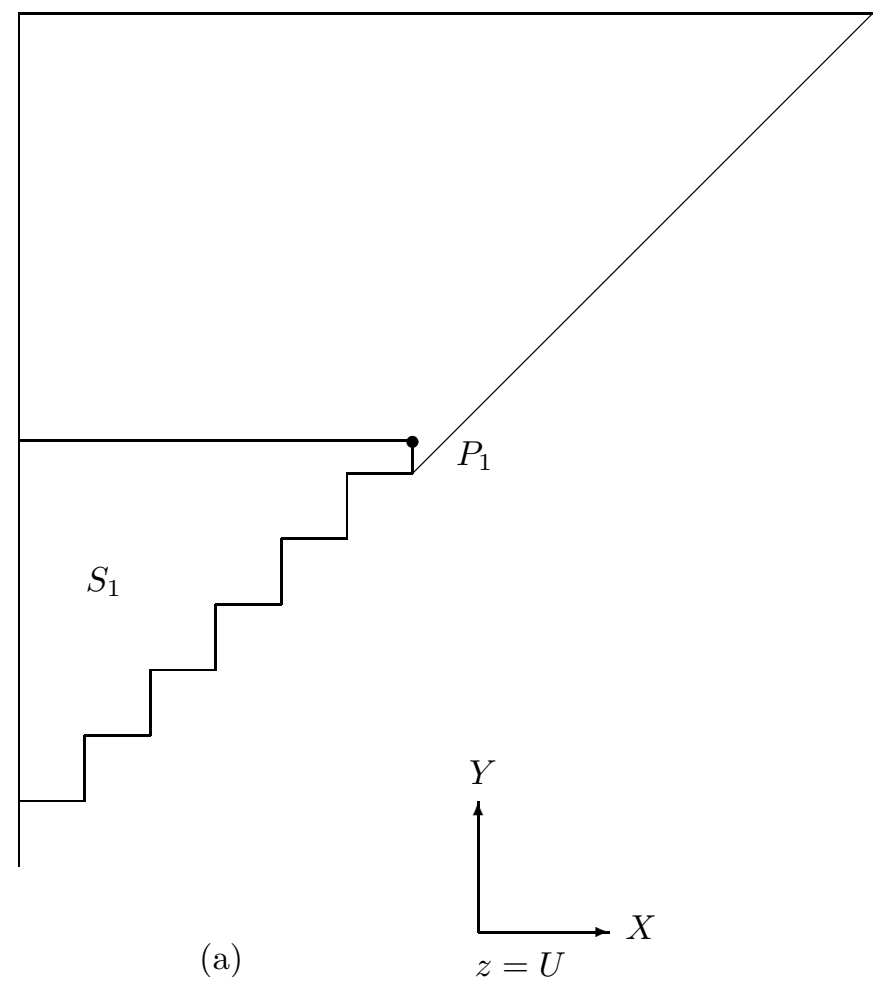

Fig. 5 (a).

Partitioning $S_{3}$ into a rectangle $S_{3}^{\prime}$ and a (2-dimensional) cone $S_{3}^{\prime}$, we obtain

$$
\begin{gathered}
\left\|S_{3}\right\|=\frac{1}{2}\left(\lceil u\rceil-1+v_{1}+U+u\right) \mu\left(S_{3}^{\prime}\right)+(U+\lceil u\rceil-1) \mu\left(S_{3}^{\prime \prime}\right), \\
\mu\left(S_{3}^{\prime}\right)=\left(\lceil u\rceil-1-v_{1}\right)(U-u), \mu\left(S_{3}^{\prime \prime}\right)=\left(\begin{array}{c}
U-(\lceil u\rceil-1) \\
2
\end{array}\right),
\end{gathered}
$$

and

$$
\mu\left(S_{3}\right)=\mu\left(S_{3}^{\prime}\right)+\mu\left(S_{3}^{\prime \prime}\right)
$$

(see Figure 5 (c).) Thus, it follows from (7.12) - (7.16) that

$$
\frac{1}{2}\left[U-u-(\lceil u\rceil-1)+v_{1}\right]\left(\lceil u\rceil-1-v_{1}\right)(U-u)-\left(\lceil u\rceil-1-v_{1}\right)\left(\begin{array}{c}
U-(\lceil u\rceil-1) \\
2
\end{array}\right)+\eta \mu\left(S_{3}\right) \geq 0 .
$$

(7.11) and (7.17) imply

$$
\eta \mu\left(S_{3}\right)>\left(\lceil u\rceil-1-v_{1}\right)\left(\begin{array}{c}
U-(\lceil u\rceil-1) \\
2
\end{array}\right) .
$$




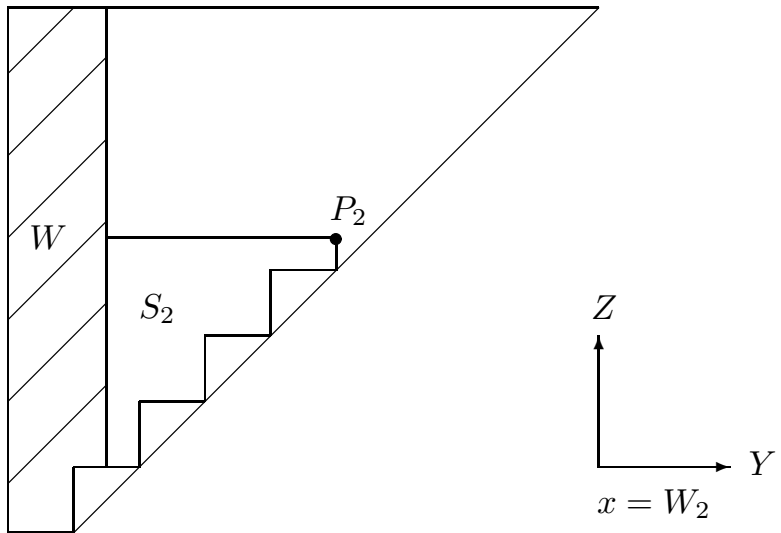

(b)

Fig. 5 (b).

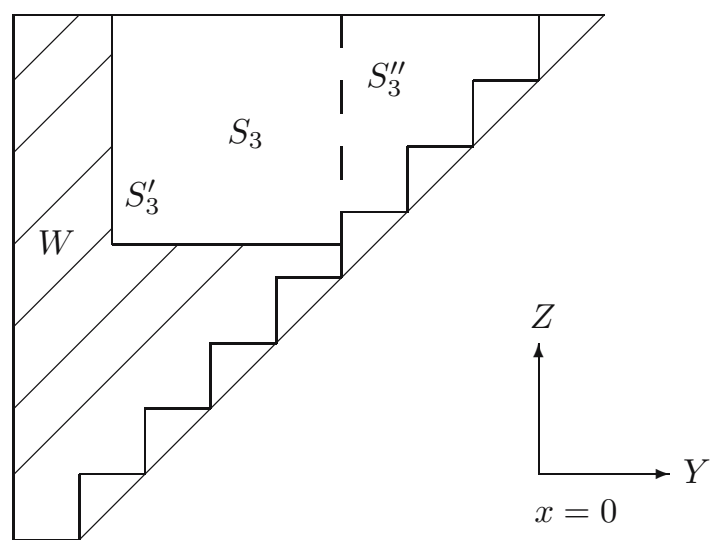

(c)

Fig. 5 (c).

However, by (7.15) and (7.16)

$$
\frac{\mu\left(S_{3}\right)}{\left(\lceil u\rceil-1-v_{1}\right)\left(\begin{array}{c}
U-(\lceil u\rceil-1) \\
2
\end{array}\right)}=\frac{U-u}{\left(\begin{array}{c}
U-(\lceil u\rceil-1) \\
2
\end{array}\right)}+\frac{1}{\lceil u\rceil-1-v_{1}} \leq 4 \text {, if } U-(\lceil u\rceil-1) \geq 2 .
$$

On the other hand, by the definition of $\eta, \eta \leq \frac{1}{4}$, which contradicts (7.18) and (7.19). When $U-\lceil u\rceil-1 \leq 1$, we can directly derive a contradiction.

Thus we are left with the case $w_{1}<\left\lceil v_{1}\right\rceil-1$ (and $\left\lceil v_{1}\right\rceil<u$ ), i.e. both of the regular $X$ - and $Y$-surfaces pass through $P_{1}$, or in other words neither of the 
surfaces passes through $P_{2}$ unless $P_{2}$ shares one of them with $P_{1}$. In fact, all of the following 3 subcases are not new to us.

Subcase 2.4: There is no regular surface passing through $P_{2}$, i.e. $P_{2}=(\lceil u\rceil-$ $2,\lceil u\rceil-1, u)$. Then the top part of $W$, namely, $W_{t} \triangleq W \cap\{(x, y, z): z>u\}$ is a cylinder with a 2 dimensional trapezoid $R_{2}\left(w_{1}, v_{1}\right)$ (its irregular $Z$-surface) as base. By similar reasoning with Lemma 5 as after (7.11) we can assume $v_{1}=\lfloor u\rfloor$, which has been treated in the subcase 2.1.

Subcase 2.5: $P_{1}$ and $P_{2}$ share a regular $X$-surface, i.e. $w_{1}=w_{2}$ and $v_{2}=\lceil u\rceil-1$. Then $\hat{W}^{(U)}$ falls into subcase 2.4 .

Subcase 2.6: $P_{1}$ and $P_{2}$ share a regular $Z$-surface, i.e. $v_{1}=v_{2}$, and $w_{2}=$ $\left\lceil v_{2}\right\rceil-1$. Then $\hat{W}^{(U)}$ falls into subcase 2.3 .

\section{A Last Auxiliary Result}

Lemma 8. For $U \in \mathbb{Z}^{+}, U \geq 6, \alpha=\left(\begin{array}{c}U \\ 3\end{array}\right)-\left(\begin{array}{c}m \\ 3\end{array}\right)<\frac{1}{2}\left(\begin{array}{c}U \\ 3\end{array}\right)$ and $m \in \mathbb{Z}^{+}$

$$
\left\|R\left(V_{\alpha}(U), U\right)\right\|>\|K(u)\|, \quad \text { if } \mu(K(u))=\alpha=\mu\left(R_{\alpha}(U), U\right) .
$$

Proof. At first let us restrict ourselves to $U \geq 12$. We know from (i) in Lemma 4 that

$$
6 \mu(K(u))=6\left(\begin{array}{c}
\lfloor u\rfloor \\
3
\end{array}\right)+6 \theta\left(\begin{array}{c}
\lfloor u\rfloor \\
2
\end{array}\right)=(u-1)^{3}-\left\{\left[3\left(\theta-\frac{1}{2}\right)^{2}+\frac{1}{4}\right]\lfloor u\rfloor-(1-\theta)^{3}\right\}
$$

Therefore,

$$
d(K(u)) \geq \frac{3}{2} u>\frac{3}{2}\left[6 \mu(K(u))^{\frac{1}{3}}+1\right]=\frac{3}{2}\left[(6 \alpha)^{\frac{1}{3}}+1\right] .
$$

On the other hand for $\eta>0$, by (8.2)

$$
\begin{gathered}
{[u-(1+\eta)]^{3}=(u-1)^{3}-3 \eta(u-1)^{2}+3 \eta^{2}-\eta^{3}} \\
=6 \mu(K(u))-\left[3 \eta(u-1)^{2}-3 \eta^{2}(u-1)+\eta^{3}-\left[3\left(\theta-\frac{1}{2}\right)^{2}+\frac{1}{4}\right]\lfloor u\rfloor+(1-\theta)^{3}+\eta^{3}\right] \\
=6 \mu(K(u))-\left[3 \eta\lfloor u\rfloor^{2}-3\left(2 \eta \bar{\theta}+\eta^{2}-\bar{\theta}(1-\bar{\theta})+\frac{1}{3}\right)\lfloor u\rfloor+(\bar{\theta}+\eta)^{3}\right] \\
\leq 6 \mu(K(u))-3\lfloor u\rfloor\left[\lfloor u\rfloor \eta-\left(2 \eta+\eta^{2}+\frac{1}{3}\right)\right], \\
\text { where } \bar{\theta} \triangleq 1-\theta .
\end{gathered}
$$

Let

$$
\eta=\xi-\frac{2 \theta \bar{\theta}}{(\lfloor u\rfloor-2)+6 \theta}>0
$$

and $\eta, \xi$ will be defined later. Then by (8.4) and (8.5), 


$$
d(K(u)) \leq \frac{3}{2}\left[6 \mu(K(u))^{\frac{1}{3}}+(1+\xi)\right]
$$

when

$$
\lfloor u\rfloor \geq \frac{2 \eta+\eta^{2}+\frac{1}{3}}{\eta},
$$

Choose $\xi_{1}=0.12$ and $\xi_{2}=0.035$, to estimate $d(K(u))$ and $d(K(U))$, resp. By our assumption $u \geq 7 \frac{20}{29}$, if $U=12$, and $u>8$, if $U \geq 13$. Then one can verify (8.6) with (8.5) for $u, \xi_{1}$ (or $\left.U, \xi_{2}\right)$. So, by (8.7)

$$
\begin{aligned}
& d(K(u)) \leq \frac{3}{2}\left[(6 \mu(u))^{\frac{1}{3}}+1+\xi_{1}\right]=\frac{3}{2}\left((6 \alpha)^{\frac{1}{3}}+1+\xi_{1}\right) \\
& d(K(U)) \leq \frac{3}{2}\left[(6 \mu(K(U)))^{\frac{1}{3}}+1+\xi_{2}\right] .
\end{aligned}
$$

Setting $\alpha=\lambda \mu(K(U))$, by Lemmas 3 and 4 , (8.3), and (8.8), we obtain

$$
\begin{gathered}
\left\|R\left(V_{\alpha}(U), U\right)\right\|-\|K(u)\|=\frac{3}{2} U \mu(K(u))-3(\mu(K(U))-\alpha)+\left\|\hat{R}^{(U)}\left(V_{\alpha}(U), U\right)\right\|-\|K(u)\| \\
\geq \frac{3}{2}\left\{\left[(6 \mu(K(U)))^{\frac{1}{3}}+1+\xi_{2}\right](2 \alpha-\mu(K(U)))+\left[(6[\mu(K(U))-\alpha])^{\frac{1}{3}}+1\right]\right. \\
\left.\cdot(\mu(K(U))-\alpha)-\left[(6 \alpha)^{\frac{1}{3}}+1+\xi_{1}\right] \alpha\right\}=\frac{3}{2} \sqrt[3]{6} \mu(K(u)) f(\lambda), \text { where } \\
f(\lambda)=2 \lambda-1+(1-\lambda)^{\frac{1}{3}}-\lambda^{\frac{1}{3}}-\frac{\xi_{2}+\left(\xi_{1}-2 \xi_{2}\right) \lambda}{(6 \mu(K(U)))^{\frac{1}{3}}}
\end{gathered}
$$

is concave in $\lambda$. Let $\varepsilon_{1}=\frac{2.7}{(6 \mu(K(U)))^{\frac{1}{3}}}, \varepsilon_{2}=\frac{2.68 / 2^{\frac{5}{3}}}{(6 \mu(K(U)))^{\frac{1}{3}}}$ and $M \in \mathbb{Z}^{+}$be specified by

$$
\left(\begin{array}{c}
M \\
3
\end{array}\right) \leq \frac{1}{2}\left(\begin{array}{c}
U \\
3
\end{array}\right)<\left(\begin{array}{c}
M+1 \\
3
\end{array}\right)
$$

Then

$$
\varepsilon_{1}<\frac{3}{U}=\frac{\left(\begin{array}{c}
U-1 \\
2
\end{array}\right)}{\left(\begin{array}{c}
U \\
3
\end{array}\right)}=\frac{\left(\begin{array}{c}
U \\
3
\end{array}\right)-\left(\begin{array}{c}
U-1 \\
3
\end{array}\right)}{\mu(J(U))},
$$

and as $\frac{\left[2\left(\begin{array}{c}M+1 \\ 2\end{array}\right)\right]^{3}}{\left[6\left(\begin{array}{c}M \\ 3\end{array}\right)\right]^{2}}=\frac{M(M-1)}{(M+1)}$, by (8.11) and $M>9$ (when $\left.U>12\right)$,

$$
\begin{aligned}
\frac{1}{2} \frac{\left(\begin{array}{c}
M \\
2
\end{array}\right)}{\left(\begin{array}{c}
U \\
3
\end{array}\right)} & =\frac{1}{4}\left[\frac{M(M-1)}{(M+1)^{2}}\right]^{\frac{1}{3}} \frac{\left(6\left(\begin{array}{c}
M+1 \\
3
\end{array}\right)\right)^{\frac{1}{3}}}{\mu(K(U))}>\frac{3}{2}\left(\frac{1}{2}\right)^{\frac{2}{3}}\left[\frac{M(M-1)}{(M+1)}\right]^{\frac{1}{3}} \frac{1}{[6 \mu(K(U))]^{\frac{1}{3}}} \\
& \geq \frac{3}{2^{\frac{5}{3}}}(0.72)^{\frac{1}{3}} \frac{1}{[6 \mu(K(U))]^{\frac{1}{3}}}=\frac{2.68884 \ldots}{2^{\frac{5}{3}}} \frac{1}{[6 \mu(K(U))]^{\frac{1}{3}}}>\varepsilon_{2} .
\end{aligned}
$$

However, with Taylor's expansion,

$$
\begin{aligned}
& f\left(\varepsilon_{1}\right) \geq 2 \varepsilon_{1}-\frac{4}{3} \varepsilon_{1}+\frac{4}{9} \varepsilon_{1}^{2}-\varepsilon_{1}^{\frac{4}{3}}-\frac{\xi_{2}+\left(\xi_{1}-2 \xi_{2}\right) \varepsilon_{1}}{(6 \mu(K(U)))^{\frac{1}{3}}} \\
& \begin{array}{l}
=\frac{1}{[6 \mu(K(U))]^{\frac{1}{3}}}\left(\frac{2}{3} \times 2.7-2.7 \times \varepsilon_{1}^{\frac{1}{3}}-\xi_{2}\right) \\
+\frac{\varepsilon_{1}}{[6 \mu(K(U))]^{\frac{1}{3}}}\left[\frac{4}{9} \times 2.7-\left(\xi_{1}-2 \xi_{2}\right)\right]>0 .
\end{array}
\end{aligned}
$$


Moreover, set $g(x)=(1+x)^{\frac{4}{3}}-(1-x)^{\frac{4}{3}}$. Then

$$
g(0)=g^{\prime \prime}(0)=0, g^{\prime}(0)=\frac{8}{3} \text { and } g^{\prime \prime}(x)>-0.6254,
$$

when $0 \leq x \leq 2 \varepsilon_{2}<0.1551$. Thus, by the definition of $\varepsilon_{2}$ and Taylor's expansion again

$$
\begin{gathered}
f\left(\frac{1}{2}-\varepsilon_{2}\right)=-2 \varepsilon_{2}+\left(\frac{1}{2}\right)^{\frac{4}{3}} g\left(2 \varepsilon_{2}\right)-\frac{\xi_{1}\left(\frac{1}{2}-\varepsilon_{2}\right)+2 \xi_{2} \varepsilon_{2}}{[6 \mu(K(U))]^{\frac{1}{3}}} \\
\geq-\left(2 \varepsilon_{2}\right)+\left(\frac{1}{2}\right)^{\frac{4}{3}} \frac{8}{3}\left(2 \varepsilon_{2}\right)-0.6254\left(2 \varepsilon_{2}\right)^{3}-\frac{\xi_{1}\left(\frac{1}{2}-\varepsilon_{2}\right)+2 \xi_{2} \varepsilon_{2}}{[6 \mu(K(U))]^{\frac{1}{3}}} \\
=2 \varepsilon_{2}\left[-1+\frac{2^{\frac{5}{3}}}{3}-0.6254\left(2 \varepsilon_{2}\right)^{2}-\frac{2^{\frac{2}{3}}}{2.68}\left[\frac{1}{2} \xi_{1}\left(1-2 \varepsilon_{2}\right)+\xi_{2}\left(2 \varepsilon_{2}\right)\right]\right] \\
\geq 2 \varepsilon_{2}[-1+1.05826 \cdots-0.0150 \cdots-0.0332 \ldots]>0 .
\end{gathered}
$$

(8.14), (8.15) and the convexity of $f$ imply $f(\lambda)>0$, when $\lambda \in\left[\varepsilon_{1}, \frac{1}{2}-\varepsilon_{2}\right]$, or, in other words, if $U \geq 12$ and $\varepsilon_{1} \mu(K(U)) \leq \alpha \leq\left(\frac{1}{2}-\varepsilon_{2}\right) \mu(K(U))$, then $\left\|R\left(V_{\alpha}(U), U\right)\right\|>\|K(U)\|$. On the other hand (8.12) and the assumption on $\alpha$ together imply $\alpha>\varepsilon_{1} \mu(K(U))$. Moreover it follows from the assumption on $\alpha$, (8.11) and (8.13), that $\alpha \leq\left(\frac{1}{2}-\varepsilon_{2}\right) \mu(K(U))$, unless

$$
\alpha=\left(\begin{array}{c}
U \\
3
\end{array}\right)-\left(\begin{array}{c}
M+1 \\
3
\end{array}\right) \text { and }\left(\begin{array}{c}
M \\
3
\end{array}\right) \leq \alpha \leq\left(\begin{array}{c}
M+1 \\
3
\end{array}\right)
$$

where $M$ is defined by $(8.11)$.

However (8.16) implies $\hat{R}^{(U)}\left(V_{\alpha}(U), U\right)=K(M+1)$ and $u \in[M, M+1]$. Therefore

$$
\hat{R}^{(U)}\left(V_{\alpha}(U), U\right) \backslash K(u)=\{(x, y, z): u<z \leq M+1,0<x<y<M,\lceil x\rceil \neq\lceil y\rceil\} \triangleq \Delta, \text { say. }
$$

This and Lemma 4 imply

$$
d(\Delta)=M+\frac{M+1+u}{2} \geq 2 M+\frac{1}{2} .
$$

Moreover, one can easily check in our case (i.e. $U \geq 12$ ) that $M \geq \frac{3}{4} U$, which together with (8.18) means that

$$
d(\Delta)>\frac{3}{2} U
$$

This and Lemmas 3, 4 imply

$$
\begin{gathered}
\left\|R\left(V_{\alpha}(U), U\right)\right\|-\|K(U)\|=\frac{3}{2} U \mu(K(U))-\frac{3}{2} U(\mu(K(U))-\alpha) \\
+\left(\left\|\hat{R}^{(U)}\left(V_{\alpha}(U), U\right)\right\|-\|K(u)\|\right)=\frac{3}{2} U[\alpha-(\mu(K(U))-\alpha)]+\|\Delta\| \\
=\frac{3}{2} U\left(\mu\left(K(u)-\hat{R}^{(U)}\left(V_{\alpha}(U), U\right)\right)+\|\Delta\|=\left(d(\Delta)-\frac{3}{2} U\right) \mu(\Delta)>0 .\right.
\end{gathered}
$$


i.e. so far, we have shown (8.1) for $U \geq 12$. Finally, we check (8.1) directly for $U=6,7, \ldots, 11$.

Remark 3. For $U<6$, there is no room for $\alpha=\left(\begin{array}{c}U \\ 3\end{array}\right)-\left(\begin{array}{c}M \\ 2\end{array}\right)<\frac{1}{2}\left(\begin{array}{c}U \\ 3\end{array}\right)$.

\section{Main Result for $k=3$ and $\operatorname{Good} \alpha$}

Now let us return to our main problem in the discrete model. Denote by $R^{*}(v, u)$ the downset of $(v, u-1, u)\left(v, u \in \mathbb{Z}^{+}\right)$in $\mathcal{L}(U, 3)$ and by $K^{*}(u)$ the downset of $(u-2, u-1, u)\left(u \in \mathbb{Z}^{+}\right)$in $\mathcal{L}(U, 3)$. Then Lemmas 2,3 , and 8 and Theorems 1 and 2 together imply immediately this solution.

Theorem 3. Let $U \in \mathbb{Z}^{+}, U \geq 6$, then

(i) For $\alpha=\left(\begin{array}{c}U \\ 3\end{array}\right)-\left(\begin{array}{c}m \\ 3\end{array}\right) \leq \frac{\left(\begin{array}{c}U \\ 3\end{array}\right)}{2}$ for some $m \in \mathbb{Z}^{+}$, $\max _{|\mathcal{A}|=\alpha} \mathcal{P}(\mathcal{A})$ is achieved by $\mathcal{R}^{*}(U-m, U)$.

(ii) For $\alpha=\left(\begin{array}{c}m \\ 3\end{array}\right) \geq \frac{\left(\begin{array}{c}U \\ 3\end{array}\right)}{2}$ for some $m \notin \mathbb{Z}^{+}$ $\max _{|\mathcal{A}|=\alpha} \mathcal{P}(\overline{\mathcal{A}})$ is achieved by $K^{*}(m)$.

\section{A False Natural Conjecture for $k=3$ and General $\alpha$; There Is "Almost" No "Order" at All}

We conclude our paper by taking a look at general $\alpha$. Both, the result for $k=2$ in [2] and our result for $k=3$ and good $\alpha$ suggest that the following conjecture is reasonable, namely, that for $k=3$ and $\alpha$ with

$$
\left(\begin{array}{c}
U \\
3
\end{array}\right)-\left(\begin{array}{c}
a+1 \\
3
\end{array}\right)<\alpha<\left(\begin{array}{l}
U \\
3
\end{array}\right)-\left(\begin{array}{l}
a \\
3
\end{array}\right) \leq N(\alpha)<\frac{\left(\begin{array}{c}
U \\
3
\end{array}\right)}{2},
$$

where $a \in \mathbb{Z}^{+}$and $N(\alpha)$ is a function depending only on $\alpha$, if $U$ is big enough, the following configuration $W$ is optimal for maximizing $\mathcal{P}(\mathcal{A})$ :

(i) take the $\left(\begin{array}{c}U \\ 3\end{array}\right)-\left(\begin{array}{c}a+1 \\ 3\end{array}\right)$ points $(x, y, z)$ with $x \leq U-(a+1)$ in $S_{U, 3}$

(ii) add the $\alpha-\left[\left(\begin{array}{c}U \\ 3\end{array}\right)-\left(\begin{array}{c}a+1 \\ 3\end{array}\right)\right]$ points $(U-a, y, z)$ where $(y, z)$ are points of a quasi-star or a quasi-complete graph in the sense of [2] according to the value of $\alpha-\left[\left(\begin{array}{c}U \\ 3\end{array}\right)-\left(\begin{array}{c}a+1 \\ 3\end{array}\right)\right]$.

However, this conjecture, which has been made by several authors, is false.

Example 1: For $\alpha_{0} \triangleq\left[\left(\begin{array}{c}U \\ 3\end{array}\right)-\left(\begin{array}{c}U-2 \\ 3\end{array}\right)\right]-(U-2)-(U-3)=\left(\begin{array}{c}U \\ 3\end{array}\right)-\left(\begin{array}{c}U-2 \\ 3\end{array}\right)-2 U+5$ (when $U$ is big enough), theW described above is $S_{1} \backslash\left(S_{2} \cup S_{3}\right)$ where $S_{1} \triangleq$ $\left\{(x, y, z) \in S_{U, 3}: x=1,2\right\}$.

$$
S_{2} \triangleq\{(2,3, U),(2,4, U), \ldots,(2, U-2, U),(2, U-1, U)\},
$$

and $S_{3}$ is listed in (10.2) below. 
Now let us consider the configuration $W^{\prime}$ with $W^{\prime} \triangleq S_{1} \backslash\left(S_{2} \cup S_{3}^{\prime}\right)$, where $S_{3}^{\prime}$ is also listed in (10.2).

$S_{3}:(2,3, U-1),(2,4, U-1), \ldots,(2, U-2, U-1),(2, U-3, U-2),(2, U-4, U-2)$

$S_{3}^{\prime}:(1,2, U),(1,3, U),(1,4, U), \ldots,(1, U-2, U),(1, U-1, U)$.

Thus, $\left\|S_{3}\right\|>\left\|S_{3}^{\prime}\right\|$ when $U>10$ and therefore $\|W\|<\left\|W^{\prime}\right\|$. This example tells us that a solution for general $\alpha$, even when $k=3$, is much more challenging. Actually, if we pay a little bit more attention to it, we will find a deeper result just at our hands. People working on these kinds of problems usually wish to find "an order", more precisely a nested optimal sequence such as

$$
W_{1} \subset W_{2} \subset W_{3} \subset \ldots
$$

where $W_{i}$ is optimal for size $i$. It is not surprising that in many cases, obviously including our problem, there is no order at all. In these cases, and in particular for our case, we define $M_{k}$ as the maximal integer s.t. the optimal nested chain with length $M_{k}$ i.e. the optimal nested chain

$$
W_{1} \subset W_{2} \subset W_{3} \subset \cdots \subset W_{M_{k}}
$$

exists. Considering our problem we only need to study the $\alpha$-s with $\alpha \leq \frac{1}{2}\left(\begin{array}{c}U \\ 3\end{array}\right)$, because we can take "complements". Therefore we wish $M_{k}$ to be close to $\frac{1}{2}\left(\begin{array}{c}U \\ 3\end{array}\right)$. In fact in [2], it was shown that $M_{2} \geq \frac{1}{2}\left(\begin{array}{l}U \\ 2\end{array}\right)-\frac{U}{2}$, and that therefore $M_{2}$ is asymptotically equal to $\frac{1}{2}\left(\begin{array}{c}U \\ 3\end{array}\right)$ (i.e. $\frac{\frac{1}{2}\left(\begin{array}{c}U \\ 2\end{array}\right)-M_{2}}{\left(\begin{array}{c}U \\ 2\end{array}\right)} \rightarrow 0$ ).

However, it is surprising that there is a jump between $M_{2}$ and $M_{3}$, because $M_{3}$ is asymptotically close to zero as can be seen from the following result.

\section{Theorem 4.}

$$
M_{3}<\left(\begin{array}{l}
U \\
3
\end{array}\right)-\left(\begin{array}{c}
U-2 \\
3
\end{array}\right) \triangleq \alpha_{2} \text { for } U>U_{0} .
$$

Proof. Assume the result is false. Then there is a nested optimal chain $W_{1} \subset$ $W_{2} \subset \cdots \subset W_{\alpha_{2}}$.

Let $\alpha_{0}, W$ and $W^{\prime}$ be defined as in Example 1 and set $\alpha_{1} \triangleq\left(\begin{array}{c}U \\ 3\end{array}\right)-\left(\begin{array}{c}U-1 \\ 3\end{array}\right)$. Then (when $U$ is big enough) $\alpha_{1}<\alpha_{0}<\alpha_{2}$ and therefore $W_{\alpha_{1}} \subset W_{\alpha_{0}} \subset \stackrel{\sqrt[3]{W}}{\alpha_{\alpha_{2}}}$. First of all, we draw attention to the fact that in the proofs in Section 3, we actually have already proved that the optimal configurations in Theorem 3 are unique (except if $\alpha=\frac{1}{2}\left(\begin{array}{c}U \\ 3\end{array}\right)$.) Therefore, $W_{\alpha_{1}}=R^{*}(1, U)$ and $W_{\alpha_{2}}=R^{*}(2, U)$ or

$$
(1, U-1, U) \in W_{\alpha_{1}} \text { and }(2, U-1, U) \in W_{\alpha_{2}}
$$


and so

$$
(1, U-1, U) \in W_{\alpha_{0}}
$$

Consequently,

$$
W_{\alpha_{0}} \neq W^{\prime}
$$

Moreover, there exists an $\left(x_{0}, y_{0}, z_{0}\right) \in W_{\alpha_{0}}$ with $x_{0} \geq 3$, because otherwise by Theorems 2 and 3 in [2] $\left\|W_{\alpha_{0}}\right\|=\|W\|$, which would contradict Example 1 (here $W$ and $W^{\prime}$ are defined as in Example 1). However, $\left(x_{0}, y_{0}, z_{0}\right) \notin R^{*}(2, U)=$ $W_{\alpha_{2}} \supset W_{\alpha_{0}}$, a contradiction.

\section{A Related Topic: The Maximal Moments for the Family of Measurable Symmetric Downsets}

Next let us drop the condition $\lceil x\rceil \neq\lceil y\rceil,\lceil y\rceil \neq\lceil z\rceil$ used in the definition of $S_{U, 3}$ in previous sections, i.e. consider the lattice $\alpha^{\prime}(U, 3) \triangleq\left(S_{U, 3}^{\prime}, \leq\right), S_{U, 3}^{\prime} \triangleq$ $\left\{(x, y, z) \in R^{3}: 0 \leq x \leq y \leq z\right\}$. The problem becomes more smooth and therefore much simpler. To see this, we mention here two observations.

(a) To guarantee the formula analogous to (4.8), we don't have to require $u \in \mathbb{Z}^{+}$.

(b) One can simply derive a lemma analogous to Lemma 6, by standard methods in calculus (such as to take right derivatives and so on).

In fact, in a similar but much simpler way we can prove the following result.

Theorem 5. For $U \in R$ let $I_{U}=[0, U]^{3} \subset \mathbb{R}^{3}$ and let $\mathcal{F}_{\alpha}$ be the family of the Lebesgue measurable subsets $S$ of $I_{U}$, satisfying

(i) For every $S \in \mathcal{F}_{\alpha} \mu(S)=\alpha$.

(ii) For every permutation $\pi$ on $\{1,2,3\}$ and every $S \in \mathcal{F}_{\alpha}\left(x_{1}, x_{2}, x_{3}\right) \in S$ implies $x_{\pi(1)}, x_{\pi(2)}, x_{\pi(3)} \in S$.

(iii) For every $S \in \mathcal{F}_{\alpha},(x, y, z) \in S$ and $\left(x^{\prime}, y^{\prime}, z^{\prime}\right) \leq(x, y, z)$. Also $\left(x^{\prime}, y^{\prime}, z^{\prime}\right) \in$ $S$.

Then $\max _{S \in \mathcal{F}_{\alpha}}\|S\|$, where $\|S\|=\int_{S}(x+y+z) d x d y d z$, is achieved by a set $S^{*} \in \mathcal{F}_{\alpha}$ of the form

$$
S^{*}=\left\{\begin{array}{c}
\{(x, y, z): \min \{x, y, z\} \leq v\} \text { for some } v=v(\alpha), \text { if } \alpha \leq \frac{U^{3}}{2} \\
\{(x, y, z): 0 \leq x, y, z \leq u\} \text { for some } u=u(\alpha), \text { if } \alpha \geq \frac{U^{3}}{2}
\end{array}\right.
$$




\section{References}

1. R. Ahlswede and N. Cai, On edge-isoperimetric theorems for uniform hypergraphs, Preprint 93-018, SFB 343 "Diskrete Strukturen in der Mathematik", Universität Bielefeld, 1993.

2. R. Ahlswede and G. Katona, Graphs with maximal number of adjacent pairs of edges, Acta. Math. Sci. Hungaricae Tomus 32, 1-2, 97-120, 1978.

3. R. Ahlswede and G. Katona, Contributions to the geometry of Hamming spaces, Discrete Math. 17, 1-22, 1977.

4. B. Bollobás and I. Leader, Edge-isoperimetric inequalities in the grid, Combinatorica 11, 4, 299-314, 1991.

5. L.H. Harper, Optimal assignments of numbers to vertices, SIAM J. Appl. Math. 12, 131-135, 1964.

6. R. Ahlswede, Simple hypergraphs with maximal number of adjacent pairs of edges, J. Combinatorial Theory, Series B, Vol. 28, No. 2, 164-167, 1980.

7. S.L. Bezrukov and V.P. Boronin, Extremal ideals of the lattice of multisets with respect to symmetric functionals (in Russian), Diskretnaya Matematika 2, No. 1, 50-58, 1990.

8. R. Ahlswede and I. Althöfer, The asymptotic behaviour of diameters in the average, Preprint 91-099, SFB 343, Diskrete Strukturen in der Mathematik, J. Combinatorial Theory B., Vol. 61, No. 2, 167-177, 1994.

9. R. Ahlswede and N. Cai, On partitioning and packing products with rectangles, Combin. Probab. Comput. 3, no. 4, 429-434, 1994. 